\title{
INVENTARIANDO LA BIODIVERSIDAD EN EL PARQUE NACIONAL DE LA CALDERA DE TABURIENTE (LA PALMA, ISLAS CANARIAS, ESPAÑA): NOVEDADES CIENTÍFICAS
}

\author{
T. Domingo-Quero, M. A. Alonso-Zarazaga, A. Sánchez-Ruiz, R. Araujo Armero, \\ A. Navas Sánchez, S. Sánchez Moreno, R. García Becerra*, M. Nebreda, M. \\ Sánchez Ruiz, F. Fontal-Cazalla y J. L. Nieves-Aldrey
}

\begin{abstract}
RESUMEN
El presente artículo es el primer resultado de un convenio entre el Organismo Autónomo Parques Nacionales y el Consejo Superior de Investigaciones Científicas, denominado "Inventario y estudio de la Fauna Invertebrada del Parque Nacional de La Caldera de Taburiente". Se detallan las novedades faunísticas encontradas hasta el momento entre los ejemplares de invertebrados terrestres y dulceacuícolas recogidos durante los dos años de muestreo (julio de 1999 a julio de 2001), cuyo número se estima en unos 500.000. Se hace una breve descripción de la planificación y la metodología aplicadas al inventario de la fauna invertebrada y se tabulan las novedades. En su estado actual el inventario arroja 284 familias, 594 géneros y 739 especies. Para la fauna de las Islas Canarias, el registro actual de táxones nuevos es de 29 familias, 115 géneros (de los cuales 1 género ha sido confirmado como nuevo para la Ciencia y otros 3 están pendientes de confirmación) y 187 especies (de las cuales 24 nuevas para la Ciencia). Además, 242 géneros y 338 especies son nuevas para la fauna de la isla de La Palma, conociéndose con anterioridad de otras islas del archipiélago. Otros 47 táxones aún en estudio, podrían resultar asimismo nuevos para la Ciencia. Estos resultados se han alcanzado tan sólo con el estudio de una parte mínima del material, lo que subraya la necesidad de muestreos continuados y sistemáticos para evaluar la riqueza faunística de áreas poco exploradas y su posible necesidad de protección. Por tanto, cabe esperar más novedades y el inventario puede quedar significativamente incrementado cuando se revise todo el material.

Palabras clave: Biodiversidad, inventario, invertebrados, métodos de muestreo, conservación, Parque Nacional de La Caldera de Taburiente, La Palma, Islas Canarias, novedades taxonómicas y faunísticas.
\end{abstract}

\section{ABSTRACT \\ Inventorying biodiversity at National Park of La Caldera de Taburiente (La Palma, Canary Islands, Spain): scientific novelties}

This paper is the first result of an agreement between the Organismo Autónomo Parques Nacionales and the Consejo Superior de Investigaciones Científicas, entitled "Inventory and study of the Invertebrate Fauna of the National Park of La Caldera de Taburiente". A detailed account of the faunistic novelties found up to now among the specimens of terrestrial and freshwater invertebrates collected along the two years of sampling (July 1999 to July 2001), whose number is estimated in ca. 500,000 , is given. A brief description of planning and of methodology applied to the inventory of the invertebrate fauna is made and a tabulated summary of novelties is presented. The list shows at present 284 families, 594 genera and 739 species. For the Canary Islands, the present record of new taxa is 29

Departamento de Biodiversidad y Biología Evolutiva, Museo Nacional de Ciencias Naturales (CSIC), c/ José Gutiérrez Abascal, 2, E-28006 Madrid, ESPAÑA.

Excepto: * C/ San Miguel 9, 38700 - S/C de La Palma. S/C de Tenerife. Islas Canarias, ESPAÑA. 
families, 115 genera ( 1 of them confirmed as new to Science and 3 awaiting confirmation) and 187 species (24 new to Science). Moreover, 242 genera and 338 species are new to the fauna of La Palma I., being known from other islands of the archipelago. Other 47 taxa, still being studied, could be new to Science as well. These results have been reached with the study of just a minimal part of the whole material, which underlines the need for systematic, continued sampling to evaluate the faunistic richness of poorly explored areas and its possible necessity for protection. Therefore, one should expect more novelties and the inventory may increase significantly when all the material is revised.

Keywords: Biodiversity, inventory, invertebrates, sampling methods, conservation, National Park of La Caldera de Taburiente, La Palma Island, Canary Islands, taxonomic and faunistic novelties.

\section{Introducción}

Las preguntas pueden ser simples y las respuestas complejas o imposibles. Una de las preguntas más usuales que se puede hacer a un biólogo (o al gestor de un área de conservación) es: -¿Cuántas especies alberga esta área? No es científico contestar con un: -Muchas, supongo... Aunque las preguntas puedan ser tan sencillas, en estos años de desaparición acelerada de táxones cuya importancia en los ecosistemas a buen seguro ni llegamos a sospechar, las respuestas no pueden consistir en vaguedades, sino en datos fiables, obtenidos con metodología científica.

Es obvio que si se está planeando la conservación de un área determinada de terreno para preservar las especies animales que en ella viven, es necesario conocer una serie de parámetros que nos permitan valorar los esfuerzos que se deben hacer en la conservación, entre éstos, la adecuación de ciertas áreas para reservas de la naturaleza o para uso de visitantes, etc. Los parámetros que se deben conocer acerca de las especies son: número, taxonomía, abundancia, endemicidad y distribución espaciotemporal. Estos y otros datos nos permitirán establecer la posición de cada una en el ecosistema al que pertenezcan y calcular los riesgos a que puedan verse sometidas. El reto de la conservación de la biodiversidad nos lleva a un objetivo bien definido: "acelerar el inventario de la diversidad orgánica y cuantificar la cantidad de diversidad... con el fin de proporcionar criterios científicos para tomar decisiones..." (Martín-Piera, 2000: 20).

En los últimos años, la necesidad de identificar y organizar la información existente sobre la biodiversidad se ha convertido en una tarea prioritaria, siendo la base fundamental para el desarrollo de medidas de conservación de los recursos naturales (Convenio de Diversidad Biológica, Rio de Janeiro, 1992). Desde entonces, numerosos proyectos de diferente envergadura persiguen este objetivo. Un caso ejemplar en España es el proyecto BIOTA, 1levado a cabo por la Consejería de Política Territorial y Medio Ambiente (CPTMA) del Gobierno de Canarias, con la finalidad de evaluar el estado de la biodiversidad, en función del conocimiento existente, para ayudar a la consideración de la variable ambiental en los procesos decisorios de las políticas autonómicas.

Se hace, pues, necesario comenzar el conocimiento de las áreas protegidas o a proteger mediante el levantamiento de inventarios de biodiversidad. Si esto es fácil para determinados grupos taxonómicos que forman parte de ésta (debido, fundamentalmente, a su conspicuidad), resulta muchas veces de una dificultad casi insuperable obtener resultados fiables para grupos taxonómicos que no se dejan ver fácilmente en el campo.

Muchas veces estos inventarios se van completando con esfuerzos que alcanzan un nivel de confianza asintótico, pero que puede dejarnos sorprendidos con hallazgos inesperados si se modifican tan sólo las técnicas de captura. Hay que tener en cuenta además que los inventarios son precisamente las estructuras dinámicas sobre las cuales se pueden localizar elementos indicadores de la salud de la biodiversidad y, repitiendo los muestreos, recuperar información acerca de su deterioro.

El carácter oceánico de las Islas Canarias, unido a razones bioclimáticas, geológicas y biogeográficas, entre otros factores, hace que este archipiélago destaque, en el contexto internacional, por la alta tasa de biodiversidad que alberga y el elevado grado de endemicidad que poseen su flora y su fauna. Muchas de estas especies presentan en la actualidad un estado crítico de conservación, debido fundamentalmente a la incidencia de las actividades humanas sobre los frágiles ecosistemas y hábitats insulares.

El proyecto "Inventario y estudio de la Fauna Invertebrada del Parque Nacional de La Caldera de Taburiente" tiene su origen en el establecimiento de un convenio entre el Organismo Autónomo Parques Nacionales y el Consejo Superior de Investigaciones Científicas. Un equipo de zoólogos del Museo Nacional de Ciencias Naturales se dispuso a levantar 
el inventario de los invertebrados del P.N. Caldera de Taburiente de la isla de La Palma (islas Canarias) con el objetivo de facilitar a la Dirección del Parque una herramienta tan básica como ésa para la correcta gestión y protección de los recursos naturales y su compatibilización con el uso público. El equipo está formado por cuatro científicos, encargados cada uno de un tipo de muestreo determinado, dos becarios, un asesor local y otro personal de apoyo. Han colaborado además numerosos especialistas en las diversas áreas taxonómicas. El muestreo ocupó dos años: desde julio de 1999 a julio de 2001.

Durante el diseño de los muestreos y de la base de datos para la informatización de los resultados tuvimos la ocasión de discutir detalles y aspectos de ambos con nuestro compañero Fermín Martín Piera. Aparte de comentar las dificultades inherentes al acercamiento multidisciplinar que se contemplaba en el convenio, nos indicó la necesidad de estandarizar la tabulación de datos. Fruto de todo ello es el diseño de la actual base de datos del Proyecto, pensado para un uso posterior por parte de programas de análisis de biodiversidad.

Por el momento, y dado el elevado número de ejemplares obtenidos, nos es imposible ofrecer un resultado completo siquiera a niveles taxonómicos superiores. Lo que aquí se muestra es simplemente un vistazo preliminar levantando ligeramente la tapadera de "La Caldera" que hierve de vida menuda, muchas veces desconocida, como podremos comprobar en la sección de Resultados.

Con todos estos esfuerzos encauzados en la misma dirección sólo será necesaria voluntad política para que toda esta información sea utilizada para la conservación de la diversidad biológica mundial y el legado de ésta a las generaciones futuras.

\section{DESCRIPCIÓN DEL MEDIO}

Recientemente declarada Reserva Mundial de la Biosfera, la isla de la Palma está situada en el sector noroccidental del archipiélago canario y el Parque Nacional de la Caldera de Taburiente ocupa su parte central; éste se encuentra situado entre los $28^{\circ} 41^{\prime} 53^{\prime \prime}$ y $28^{\circ} 46^{\prime} 44^{\prime \prime}$ de latitud norte y entre los $17^{\circ} 49^{\prime} 55^{\prime \prime}$ y $17^{\circ} 54^{\prime} 31^{\prime \prime}$ de longitud Oeste.

En la parte más elevada de La Palma se abre la Caldera de Taburiente, de espectacular paisaje, un estratovolcán que constituye una formidable cabecera de barranco de unos $8 \mathrm{Km}$ de diámetro y cuyas paredes rebasan los $2.000 \mathrm{~m}$ de altitud, encontrándose su fondo a unos $800 \mathrm{~m}$ sobre el nivel del mar. Además de esta fisiografía tan singular, la Caldera de Taburiente también se caracteriza por la abundancia de manantiales y la existencia de un curso fluvial permanente, rasgo poco habitual dentro del archipiélago canario. Esta abrupta morfología hace que en un espacio muy pequeño se desarrollen numerosas especies rupícolas, así como comunidades pertenecientes a los pisos de vegetación supracanario y mesocanario seco.

La parte más elevada de la Caldera corresponde al piso bioclimático supracanario que se caracteriza por la abundancia del elemento arbustivo formado por Adenocarpus viscosus spartioides y Genista benehoavensis. Fitosociológicamente, esta comunidad queda englobada en la alianza Spartocytision supranubii con una asociación propia, única en este lugar: Telino benehoavensi-Adenocarpetum spartioidis. Otras especies características son Micromeria lasiophylla palmensis y Viola palmensis, existiendo además muchas especies típicas como Spartocytisus supranubius, Plantago webbii, Lactuca palmensis, Bufonia paniculata, Echium wildpretii trichosiphon, Echium gentianoides y Bencomia exstipulata. Esporádicamente pueden hallarse Erysimum scoparium, Nepeta teydea, Descurainia gilva y Juniperus cedrus. En estas zonas también pueden observarse asociaciones típicas de las comunidades rupícolas como son: Tolpis calderae, Argyranthemum haouarytheum, Pterocephalus porphyranthus, Cheirolophus teydis, Cerastium sventenii y Senecio palmensis, pertenecientes a la asociación Tolpidetum calderae (Orden Greenovietalia).

El intransitable escarpe de la Caldera destaca por su gran dimensión y extensión, constituyendo el elemento paisajístico más destacado. En él se desarrollan comunidades rupícolas caracterizadas por la abundancia de crasuláceas de los géneros Greenovia, Aeonium, Monanthes y Aichryson, junto a numerosos endemismos canarios o macaronésicos, entre los que se encuentran numerosas especies de los géneros Silene, Sideritis, Micromeria y Sonchus.

A resguardo de los vientos alisios, se desarrolla un majestuoso pinar (Pinus canariensis) que se caracteriza por su gran extensión, su buen estado de conservación y su baja diversidad florística. Pertenece a la comunidad Cytiso-Pinetea canariensis definida en la alianza Cisto-Pinion. La manifestación más xérica del pinar se corresponde con la subasociación Cistetosum englobada por la asociación Loto hillebrandii-Pinetum canariensis y, además del pino están extensamente presentes Cistus symphytifolius y Lotus hillebrandii, otras especies que completan el complejo florístico del pinar son: Echium webbii, Gonospermum canariense, Sideritis barbellata, Chamaecytisus proliferus, Teline stenopetala, Bystropogon origanifolius e 
Hypericum grandifolium. En zonas aclaradas, cercanas a los senderos es frecuente observar Pteridium aquilinum y diversas especies ruderales como pueden ser Silene vulgaris commutata, Centranthus calcitrapa y Calendula arvensis.

En el pequeño ecotono entre el seco pinar y los barranquillos, más frescos y sombríos, afectados por masas nubosas de condensación, se establecen algunos elementos de fayal-brezal (AndryaloEricetalia), siendo el brezo Erica arborea la especie más abundante. También puede observarse algún ejemplar de Myrica faya, acompañados por Pteridium aquilinum en abundancia. Esta facies se corresponde con la subasociación ericetosum de la asociación Loto hillebrandii-Pinetum canariensis.

En los barrancos más húmedos se desarrollan algunos elementos de Pruno-Lauretea: Erica arborea, Myrica faya, Salix canariensis, algún ejemplar de Persea indica, Viburnum rigidum, Cedronella canariensis y Woodwardia radicans.

Son frecuentes en fisuras rezumantes Anogramma leptophylla, Asplenium trichomanes característicos de los hábitats más húmedos de PrunoLauretea. También se pueden observar, en estos lugares, Adiantum capillus-veneris y Adiantum reniforme; Ageratina adenophora invade los puntos de agua y sus proximidades.

En las proximidades de la Zona de Acampada, la sauceda (Rubo-Salicetum canariensis) adquiere su mayor desarrollo caracterizada por el endemismo Salix canariensis; en el suelo de este bosque abundan las especies higrófilas como Equisetum ramosissimum (Santos, 1983).

En el interior del parque existen tres pequeños asentamientos humanos: las haciendas de Tenerra y Taburiente, y el Centro de Servicios del Parque, en los que hay huertos para el autoabastecimiento y en cuyas inmediaciones se desarrollan algunas especies antrópicas como tuneras, higueras y otros frutales.

\section{Material y métodos}

El objetivo del inventario era detectar la presencia del mayor número de invertebrados posible en el área de estudio. Para tal fin se llevaron a cabo durante dos años numerosos muestreos utilizando diferentes técnicas de captura con las que se han colectado más de 450.000 ejemplares. Tras la fijación, conservación y etiquetado del material se está procediendo a su separación y distribución entre los diferentes especialistas para su determinación. Las labores básicas de preparación de muestreos y análisis preliminar de muestras, así como algunas de las técnicas de extracción de las mismas, se efectuaron en el laboratorio montado a tal efecto en las instalaciones cedidas por la Dirección del Parque Nacional de la Caldera de Taburiente, que además puso a la disposición del equipo investigador la infraestructura necesaria.

Por decisión de ambas partes durante la redacción del proyecto, algunos grupos de invertebrados quedaron fuera del inventario. Entre éstos, cabe destacar el grupo de ecto- y endoparásitos de vertebrados y los protozoos.

Se identificaron los siguientes grupos de invertebrados susceptibles de muestreo:

1. Fauna fundamentalmente voladora o en suspensión aérea: para este tipo de fauna se utilizaron preferentemente el cazamariposas, las trampas de intercepción "Malaise" y las trampas de atracción "Moericke".

2. Fauna epigea y epifítica: para el muestreo de la primera se utilizó la caza a vista (levantamiento de piedras y observación directa), las trampas de caída "pitfall" y la recogida de muestras de humus bruto para embudos Berlese mediante el aparato de Winkler; para la segunda, la caza a vista, el barrido de la vegetación mediante manga entomológica y el descortezamiento de troncos.

3. Fauna subterránea: se recogieron muestras para la extracción de nematodos, se colocaron trampas "pitfall" cebadas con queso en cuevas (fauna troglobia) y se utilizaron trampas subterráneas para el muestreo del Medio Subterráneo Superficial (MSS) (García et al., 1997).

4. Fauna especializada: se efectuó recogida de ramas para la eclosión de xilófagos y depredadores de éstos en contenedores en el laboratorio; la fauna necrófaga se muestreó utilizando como cebo cadáveres de pollo; también se hicieron muestreos nocturnos de visu con linterna, se mangueó la vegetación y se utilizó una trampa de luz (Fernández Rubio, 1985).

5. Fauna dulceacuícola: se realizaron visitas estacionales a diferentes puntos de agua en los que se muestreó la fauna presente mediante diferentes técnicas básicas (Arconada, 2000), tales como el uso de un colador, el lavado de piedras y plantas acuáticas, muestreo de sedimento, etc. Una bandeja blanca y unas pinzas blandas sirvieron para colectar los ejemplares, que se conservaron en alcohol de $70^{\circ}$. También se emplearon muestreos específicos para ácaros acuáticos. 
Tabla 1.- Trampas Malaise: identificación de la trampa, ubicación, fechas de colocación y recogida y número de muestras. UTM $(500 \times 500 \mathrm{~m})$.

Table 1.- Malaise traps: trap code, locality, dates of placement and withdrawal, and number of samples. UTM grid (500 x $500 \mathrm{~m})$.

\begin{tabular}{|c|c|c|c|c|}
\hline Trampa & Ubicación & Fecha de colocación & Fecha de recogida & Número de muestras \\
\hline M6 & $\begin{array}{l}\text { Barranco de las Traves } \\
\text { UTM 28RBS1780-2 } \\
1068 \mathrm{~m}\end{array}$ & 30 julio 1999 & 25 septiembre 2000 & 53 \\
\hline M59 & $\begin{array}{l}\text { Playa de Taburiente } \\
\text { UTM 28RBS1980-1 } \\
750 \mathrm{~m}\end{array}$ & 30 julio 1999 & 25 septiembre 2000 & 53 \\
\hline $\begin{array}{l}\text { M } \\
49 \\
50\end{array}$ & $\begin{array}{l}\text { Lomo de las Chozas } \\
\text { UTM 28RBS2073-3 } \\
1297 \text { m (31-VII-1999/12-I-2000) } \\
1277 \text { m (12-I-2000/27-IX-2000) }\end{array}$ & 31 julio 1999 & 27 septiembre 2000 & 50 \\
\hline M63 & $\begin{array}{l}\text { Parcela de los Helianthemum } \\
\text { UTM 28RBS2177-1 } \\
1377 \mathrm{~m}\end{array}$ & 31 julio 1999 & 27 septiembre 2000 & 49 \\
\hline \multirow[t]{2}{*}{ M67 } & $\begin{array}{l}\text { Parcela del Morro de la Cebolla } \\
\text { UTM 28RBS2084-3 } \\
2250 \mathrm{~m}\end{array}$ & $\begin{array}{l}22 \text { julio } 2000 \\
14 \text { marzo } 2001\end{array}$ & $\begin{array}{c}26 \text { octubre } 2000 \\
15 \text { julio } 2001\end{array}$ & $\begin{array}{l}13 \\
18\end{array}$ \\
\hline & & & TOTAL DE MUESTRAS & 236 \\
\hline
\end{tabular}

Para una información general sobre los métodos de muestreo empleados, véase Marcos-García (1988). A continuación se especifican los datos relativos a algunos métodos de muestreo y sus condiciones de uso:

- Trampas "Malaise": se pretendió muestrear la fauna voladora de cinco hábitats seleccionados como más representativos del Parque Nacional (localidades 6, 49-50, 59, 63 y 67 de la Tabla 2). Las trampas eran del modelo Townes de origen comercial (Townes, 1972; Nieves-Aldrey y Rey del Castillo, 1991), empleándose en todos los casos el modelo de luz de malla fina y color enteramente negro. Para los periodos de colocación y ubicación exacta, y el número de muestras recogidas, véase la Tabla 1. En el caso de la trampa M67, se debió interrumpir su presencia debido a las intensas precipitaciones y fuertes vientos que se producen durante el periodo invernal en esta localidad. La recogida de muestras se realizó semanalmente (salvo alguna excepción puntual), utilizándose alcohol de $70^{\circ}$ como líquido conservante.

- Trampas "Moericke": se colocó un conjunto de cuatro trampas "Moericke" amarillas situadas debajo de la pared media de cada trampa "Malaise". Cada trampa consistía en un barreño cuadrado de plástico amarillo de $35 \mathrm{~cm}$ de lado, eligiéndose este color porque es el que proporciona una mayor diversidad de especies (OrtizSánchez y Aguirre-Segura, 1993). Las trampas actuaron durante los mismos periodos, con la misma frecuencia y en las mismas localidades que las trampas "Malaise", como líquido conservante se utilizó anticongelante comercial (solución de etilenglicol) al 50\% con unas gotas de jabón. Este método se puede considerar un sumatorio entre las trampas de intercepción y las trampas atrayentes (Moericke), capturando así por una parte la fauna que tiene una capacidad de vuelo menor o que se deja caer cuando topa con un obstáculo y por otra, la fauna que resulta atraída por el color amarillo (G. Gibson, com. pers. 1999).

- Trampas de caída ("pitfall”): estas trampas se utilizaron en las mismas localidades y durante periodos no siempre coincidentes con los de las trampas Malaise. Los recipientes empleados tenían un diámetro de $6 \mathrm{~cm}$ y un fondo de $13 \mathrm{~cm}$ y se colocaron enterrados con la boca a ras de suelo, a una distancia aproximada de un metro de las trampas Malaise. Se taparon con una piedra sostenida sobre otras tres menores, dejando una distancia entre la piedra y el suelo de dos a tres $\mathrm{cm}$. La recogida de muestras se realizó semanalmente y se utilizó el mismo líquido conservante que en las trampas "Moericke". 
- Tabla 2.- Localidades de muestreo donde han aparecido novedades, indicando altitud y UTM (500 x $500 \mathrm{~m}$, excepto $\mathrm{n}^{\circ} 43,1 \times$ $1 \mathrm{Km}$ ). La provincia es Santa Cruz de Tenerife y el municipio El Paso en todas ellas excepto para la localidad $\mathrm{n}^{\circ} 43$ que corresponde al municipio de Puntallana.

Table 2.- Localities of sampling where novelties have appeared, quoting heigth and UTM grid (500 x $500 \mathrm{~m}$, excepting nr. 43,1 $\mathrm{x} 1 \mathrm{Km}$ ). All records belong to the province of Santa Cruz de Tenerife and to the municipality of El Paso, excepting locality nr. 43 which belongs to the municipality of Puntallana.

\begin{tabular}{|c|c|c|c|}
\hline $\mathbf{N}^{\mathbf{o}}$ & Localidad & Altitud (m) & UTM \\
\hline 1 & Arroyo Almendro Amargo (antes de juntarse con Rivanceras) & 470 & 28RBS1879-4 \\
\hline 2 & Arroyo de la Cumbrecita & 1200 & 28RBS2078-4 \\
\hline 3 & Arroyo Rivanceras (antes de juntarse con Almendro Amargo) & 480 & 28RBS1879-4 \\
\hline 4 & Barranco Bombas de Agua Maragaño & 2250 & 28RBS1783-2 \\
\hline 5 & Barranco de Altaguna, parcela & 2150 & 28RBS2382-2 \\
\hline 6 & Barranco de Las Traves & 1068 & 28RBS1780-2 \\
\hline 7 & Barranco de las Traves & 1050 & 28RBS1780-4 \\
\hline 8 & Barranco de las Traves & 1040 & 28RBS1780-4 \\
\hline 9 & Barranco de Tajodeque & 2100 & 28RBS1681-3 \\
\hline 10 & Barranco del Ciempiés & 930 & 28RBS1779-1 \\
\hline 11 & Barranco del Limonero & 2100 & 28RBS2280-4 \\
\hline 12 & Barranco Madera García & 1152 & 28RBS1977-3 \\
\hline 13 & Bejenado & 1410 & 28RBS1976-1 \\
\hline 14 & Bejenado, cara sureste (subida) & 1480 & 28RBS2076-1 \\
\hline 15 & Bejenado, comienzo de la senda de subida & 1300 & 28RBS2076-2 \\
\hline 16 & Bejenado; El Rodeo & 1600 & 28RBS1976-3 \\
\hline 17 & Bejenado; Pista Ferrer-Cueva de los Arenales & 1175 & 28RBS2176-2 \\
\hline 18 & Cabecera del barranco de Altaguna & 2000 & 28RBS2382-2 \\
\hline 19 & Cabecera del barranco de Las Grajas, Barranco de las Violetas & 2320 & 28RBS1784-4 \\
\hline 20 & Cantos de Turugumay (cerca de la galería) & 1230 & 28RBS1983-3 \\
\hline 21 & Carretera de la Cumbrecita; prado con taginaste azul & 1200 & 28RBS2177-1 \\
\hline 22 & Cueva de los Arenales & 1175 & 28RBS2176-2 \\
\hline 23 & Cueva de los Laberintos & 980 & 28RBS2175-1 \\
\hline 24 & Cueva de los Laberintos (junto a la entrada) & 980 & 28RBS2175-1 \\
\hline 25 & Cueva de los Sorprendidos & 1270 & 28RBS2076-4 \\
\hline 26 & Cueva de los Sorprendidos (junto a la entrada) & 1259 & 28RBS1976-2 \\
\hline 27 & Cumbrecita; Fuente de la Faya & 1100 & 28RBS2176-4 \\
\hline 28 & Degollada de las Palomas & 2210 & 28RBS1682-3 \\
\hline 29 & Dos Aguas & 450 & 28RBS1878-3 \\
\hline 30 & El Capadero (antes de llegar) & 780 & 28RBS1980-2 \\
\hline 31 & El Reventón & 538 & 28RBS1979-1 \\
\hline 32 & El Riachuelo & 950 & 28RBS2175-4 \\
\hline 33 & Fuente de la Faya & 898 & 28RBS1880-2 \\
\hline 34 & Fuente de la Zarza & 1248 & 28RBS1977-1 \\
\hline 35 & Fuente de los Gatos & 1300 & 28RBS1877-3 \\
\hline 36 & Fuente del Barranco del Limonero & 1349 & 28RBS2180-3 \\
\hline 37 & Fuente del Barranco Madera García & 1152 & 28RBS1977-3 \\
\hline 38 & Fuente del Viñátigo & 1139 & 28RBS2082-4 \\
\hline 39 & Fuente Piedra Majorera & 1410 & 28RBS2281-2 \\
\hline 40 & Fuente Prieta & 1430 & 28RBS2181-4 \\
\hline 41 & Genebuque & 1150 & 28RBS1680-2 \\
\hline 42 & Hacienda del Cura & 620 & 28RBS1577-1 \\
\hline 43 & Hoya de Lucía (cabecera del Barranco Hondo) & 2100 & 28RBS2382 \\
\hline 44 & Hoyo Verde & 1450 & 28RBS1882-3 \\
\hline 45 & La Desfondada & 1020 & 28RBS1981-1 \\
\hline 46 & Llano de los Pinos Chicos & 800 & 28RBS1880-2 \\
\hline 47 & Lomo al final de la Pista de Ferrer & 1460 & 28RBS1976-2 \\
\hline 48 & Lomo Cumplido & 1400 & 28RBS2181-3 \\
\hline 49 & Lomo de las Chozas & 1297 & 28RBS2077-3 \\
\hline 50 & Lomo de las Chozas & 1277 & 28RBS2077-3 \\
\hline 51 & Lomo Gazmil; cementerio guanche & 950 & 28RBS1981-3 \\
\hline 52 & Lomo Gazmil, ladera & 870 & 28RBS1981-3 \\
\hline 53 & Lomo Gazmil, base & 850 & 28RBS1981-3 \\
\hline 54 & Morro Colorado & 869 & 28RBS1880-1 \\
\hline 55 & Morro de los Gatos & 1353 & 28RBS1878-4 \\
\hline 56 & Parcela de Garome & 2130 & 28RBS1682-3 \\
\hline 57 & Pico de la Cruz & 2345 & 28RBS2183-1 \\
\hline
\end{tabular}


Pico de la Nieve

2230

750

730

1225

1300

1377

1050

2415

2270

2250

2250

2150

1920

835

1160

1130

1090

1050

2100
28RBS2381-2

28RBS1980-1

28RBS1980-1

28RBS2077-1

28RBS1781-3

28RBS2177-1

28RBS1881-3

28RBS1884-2

28RBS2084-3

28RBS2084-3

28RBS1785-2

28RBS1885-2

28RBS1580-4

28RBS1880-3

28RBS1680-4

28RBS1780-2

28RBS1780-2

28RBS1680-4

28RBS1682-4

28RBS1980-1
Trampas para fauna cavernícola: se utilizaron trampas de caída cebadas con queso (García et al., 1997) en diferentes puntos de tres tubos volcánicos pertenecientes a las localidades 22 , 23 y 25 del área de estudio (ver Tabla 2). La recogida de muestras se realizó mensualmente y sin interrupción durante 14 meses desde septiembre de 1999 hasta octubre de 2000 (García et al., 2002). Como líquido conservante se utilizó anticongelante con unas gotas de jabón fungicida.

- Trampas para fauna del MSS: se emplearon trampas para fauna del MSS (García et al., 1997) en tres localidades del área de estudio, muestreando ininterrumpidamente el periodo comprendido entre diciembre de 1999 y agosto de 2001. La recogida de muestras se efectuó mensualmente. Las trampas contenían una solución acuosa hipersaturada de sal como líquido conservante. Los ejemplares se lavaron en el laboratorio y se conservaron en alcohol de $70^{\circ}$.

- Muestreo de Nematoda: en determinados puntos, seleccionados previamente, se procedió a la recogida de una muestra de suelo de aproximadamente $500 \mathrm{~g}$ con un grado de humedad adecuado que contenía rizosfera. La extracción de los nematodos se llevó a cabo según el método de extracción por centrifugación en azúcar (De Grisse, 1969; Hernández y Pastor, 1989), se realizaron preparaciones semipermanentes en TAF (De Grisse, 1974) para la observación y determinación de nematodos hasta nivel de género en microscopio óptico.
- Muestreo de Tardigrada: hasta el momento no existe precedente de muestreos de tardígrados en la isla de La Palma y, a tal efecto, se recolectaron algunos musgos, líquenes y hepáticas de diferentes localidades del área de estudio. Posteriormente en el laboratorio se procedió a su extracción mediante lavado con agua destilada y tamizado con luz de malla de 1 y $0,08 \mathrm{~mm}$. La fijación se realizó con líquido de Carnoy y, para el montaje permanente se utilizó líquido de Faure; para la identificación se utilizó el microscopio óptico (Ramazzotti y Maucci, 1983).

- Muestreos de fauna acuática: además de los muestreos programados, en el periodo comprendido entre el 22 y el 24 de marzo de 2001 el Dr. Antonio García Valdecasas llevó a cabo muestreos específicos para ácaros acuáticos en diferentes localidades, por cuenta del proyecto. Se tomaron muestras en manantiales, musgos húmedos, arroyos, pequeñas cascadas y se utilizó el método de Karaman-Chappuis (un hoyo cerca de una corriente de agua, constantemente llenada con agua intersticial) (Valdecasas, 2002). Las muestras fueron lavadas utilizando un tamiz de 0,25 mm y fijadas con líquido de Angelier (Valdecasas \& Baltanás, 1989). En el laboratorio, se lavaron de nuevo y finalmente los ácaros fueron clasificados y conservados en líquido de Koenike antes de su disección.

- Muestreo de fauna necrófaga: se procedió al muestreo de la necrofauna presente en las localidades 50 y 69 (ver Tabla 2) durante el periodo comprendido entre el 14 de junio y el 2 de agosto de 2001 mediante la utilización de cadáveres 
de pollo que actuaron como cebo (Juan de Ferrer, com. pers., 2000). Se realizaron visitas semanales a los mismos, colectando la fauna que había sido atraída y procediendo a recoger el sedimento que estaba bajo el cebo. Se efectuó la crianza de las larvas hasta el desarrollo de la fase adulta.

- Mangueo de vegetación: durante dos años se hicieron diferentes recorridos en los que se muestreó la vegetación efectuando barrido con manga entomológica de especies vegetales escogidas, capturándose los ejemplares mediante un aspirador entomológico.

- Muestreo mediante técnica de Winkler-Berlese: se utiliza esta técnica para la obtención de microfauna epiedáfica y del humus bruto. Se recogieron muestras de aproximadamente $500 \mathrm{~g}$ de hojarasca y primeros centímetros de suelo en algunas localidades y se procedió in situ a la separación de la muestra por tamaños mediante el aparato de Winkler, tras lo cual el extracto de menor tamaño se trasladaba a embudos Berlese en el laboratorio para la obtención del material.

Las localidades de obtención de las muestras que han aportado alguna novedad presentada en el apartado siguiente se exponen en la Tabla 2.

\section{Resultados}

Hasta el momento de redactar este artículo se han separado y enviado a determinación aproximadamente 32.000 ejemplares de los más de 450.000 ejemplares capturados durante los muestreos. Actualmente se tienen determinaciones de $9.810 \mathrm{y}$ 7.997 ejemplares hasta nivel de género y especie respectivamente. Se han encontrado 820 especies, la mayoría resultan novedosas para el área de estudio, escasamente muestreada con anterioridad debido a su inaccesibilidad. Sin embargo, en este trabajo, sólo se han contemplado como novedosas aquellas especies de las que no se tenía constancia de su presencia en La Palma.

En la toma de decisión para la inclusión de las especies presentes en este listado se ha utilizado como información de referencia el Catálogo de los Diptera de España, Portugal y Andorra (CarlesTolrá et al., 2002) y la aún incompleta Lista de Especies Silvestres de Canarias (Izquierdo et al., 2001). Esta última no contempla los phyla Tardigrada, Platyhelminthes y Nematoda, grupos muy poco estudiados en el archipiélago, y para su inclusión en el presente trabajo se ha consultado con especialistas en esta materia. También se ha extraído información de algunos artículos de los que se tenía conocimiento por haber realizado, dentro del mismo proyecto, una base de datos de referencias bibliográficas sobre la fauna invertebrada canaria en general y de La Palma en particular, intentando así incluir en este trabajo sólo aquellas especies que no habían sido citadas con anterioridad en La Palma y en Canarias. A nuestro entender, todas las especies citadas en este artículo resultan novedosas para La Caldera de Taburiente. Se han incluido, evidentemente, las especies descritas o citadas con anterioridad cuyos materiales originales, en todo o en parte, provienen de este Proyecto (véanse Monserrat, 2002; Koponen y Askew, 2002; Valdecasas, 2002; Carles-Tolrá et al., 2002).

Se ha seguido en las categorías taxonómicas por encima de la familia, la ordenación propuesta por Southey (1978) para el phylum Nematoda, la propuesta por Ramazzotti \& Maucci (1983) para el phylum Tardigrada y la propuesta por Izquierdo et al. (2001) para el resto de phyla, mientras que las familias y sus táxones subordinados van por estricto orden alfabético.

Se exponen en el Apéndice todos los táxones que resultan novedad para La Palma, para Canarias y para la Ciencia, especificando su calificación, y a continuación las localidades donde han sido obtenidos, haciendo referencia a la fenología y al método de captura.

Para la presentación de resultados en el presente trabajo se ha procedido a la codificación de las localidades de cada especie de una forma estandarizada para que la traducción del código utilizado sea lo más intuitiva posible. Así, en los datos consta primero el tipo de muestreo utilizado (véase leyenda), seguido del número de la localidad (ver Tabla 2) y a continuación, separado por un guión y en números romanos el mes en el que han sido capturados los ejemplares.

Los ejemplares determinados tan sólo hasta nivel de género se han calificado como novedad de tipo genérico y específico porque resulta obvio que si el género es novedoso, la especie tiene que serlo a la fuerza. Sólo se han enumerado las localidades de géneros y especies; si un taxon de orden superior se considera novedoso, hay que asignarle las localidades de los táxones contenidos en él.

Por otro lado, hay especies a las que se les ha añadido la calificación "cf." (confert). Esto es así porque, en opinión de los especialistas que las han identificado, no coinciden exactamente con la especie a la que han sido asignadas. Se quiere con ello 
señalar la posibilidad de que, a posteriori, se las considere tanto miembros de la especie indicada (por tratarse de variabilidad propia de la especie a la que han sido asignadas) como que sean descritas como nuevas especies o subespecies cuando se obtengan datos que lo confirmen.

\section{Conclusiones}

En el presente trabajo se han detectado:

- 29 familias nuevas para la fauna canaria;

- 1 género nuevo para la Ciencia confirmado y 3 por confirmar;

- 115 géneros nuevos para la fauna canaria;

- 242 géneros nuevos para la fauna de La Palma;

- 24 especies nuevas para la Ciencia;

- 187 especies nuevas para la fauna canaria;

- 338 especies nuevas para la fauna de La Palma;

- 47 táxones probablemente nuevos pendientes de confirmación.

Estos datos corresponden al estudio de un porcentaje muy bajo de las muestras. De esto se pueden deducir los siguientes puntos:

El conocimiento de la fauna invertebrada del P. $\mathrm{N}$. de La Caldera de Taburiente era hasta el momento muy bajo, por lo que no resulta extraño el número de novedades para el Parque Nacional. Sin embargo, sí resulta llamativo el número de especies nuevas para la Isla y el conjunto del Archipiélago, lo que nos indica que el conocimiento de la biodiversidad canaria es aún escaso.

Además de los trabajos de recopilación bibliográfica habría que llevar a cabo inventarios estratégicamente diseñados en áreas poco exploradas para una aproximación más exacta de la distribución de la biodiversidad que existe en el Archipiélago (lo cual es además aplicable a todo el planeta), sobre todo en lugares gravemente amenazados por la actividad humana.

Observamos que el impedimento taxonómico (Wilson, 1985; Ramsay, 1986) puede ser uno de los principales motivos para este bajo nivel de conocimiento, debido, entre otras razones, aunque de índole menor, al escaso interés que se muestra en la enseñanza superior por las cuestiones de Taxonomía zoológica y botánica y al desprecio patente por estas actividades por parte de la Administración y de las personas encargadas de proporcionar puestos de trabajo relacionados con el inventario de nuestra biodiversidad y de legislar a diferentes escalas para proporcionar medios adecuados a tal fin. En resumen, queda demasiado trabajo por hacer y pocas personas preparadas para realizarlo y con escasos medios materiales. Como consecuencia de estos planteamientos erróneos de los responsables de la salvaguarda de nuestro patrimonio biológico, el "impacto científico" del trabajo taxonómico está poco remunerado social y materialmente, una traba más para el desarrollo de este tipo de estudios.

Está claro que el desarrollo de inventarios de biodiversidad implicaría un gran esfuerzo de medios humanos y económicos, pero a largo plazo supondría una inversión de futuro altamente rentable ya que permitiría dilucidar la riqueza que podríamos estar perdiendo.

Estos inventarios, una vez completos, permitirían hacer un seguimiento del medio y detectar las variaciones en el número de especies o en su abundancia, como síntoma de una mala gestión medioambiental o de un deterioro no detectado de los hábitats, y poner remedio a los desencadenantes de tal situación.

Las personas implicadas en este proyecto multidisciplinario pensamos que los resultados que aquí mostramos son, como queda patente, sólo la punta del iceberg y que el inventario completo será capaz de arrojar mucha más luz sobre el pasado, presente y futuro de una gran cantidad de interrogantes ecológicos y evolutivos acerca de la biota canaria y de las técnicas de gestión más adecuadas para mantenerla lo más intacta posible.

\section{AGRADECIMIENTOS}

Agradecemos al personal del Parque Nacional de la Caldera de Taburiente, encabezado por su Director D. Ángel Palomares Martínez, su apoyo incondicional en todas las actividades que el equipo de investigación tuvo que emprender; demostraron siempre un ejemplar conocimiento de campo del medio natural los guardas Eduardo Pérez Cáceres, Manuel Martín Pérez y Ángel Rebolé Beaumont. Asimismo quede patente nuestro agradecimiento a todos los taxónomos especialistas por su desinteresada labor en la identificación de los ejemplares, y a los Conservadores del Museo Nacional de Ciencias Naturales (CSIC) por la ayuda moral y material prestada en todo momento. Asimismo agradecemos sus indicaciones sobre los métodos de muestreo al Dr. Gary Gibson (Agriculture and Agri-Food Canada, Ottawa, Canada) y a D. Juan de Ferrer Andreu (Algeciras, España).

\section{Referencias}

ArconadA, B., 2000. Contribución al conocimiento sistemático y filogenético de la Familia Hydrobiidae 
(Mollusca, Prosobranchia) de la península ibérica. Tesis Doctoral. Universidad Autónoma de Madrid. $447 \mathrm{pp}$.

Carles-Tolrá, M. (ed.), 2002. Catálogo de los Diptera de España, Portugal y Andorra (Insecta). Monografías de la S.E.A., 8: 1-323.

Convenio de Diversidad Biológica, Rio de Janeiro, 1992. Recurso electrónico:

http://www.biodiv.org/convention/articles.asp?lg=1 (actualizado el 11 de diciembre de 2002).

De Grisse A.T., 1969. Redescription ou modification de quelques techniques utilisées dans l'étude des nematodes phytoparasitaires. Mededelingen van den Rijksfaculteit Landbouwwetenschappen be Gent, 35: 41-63.

DE GRISSE A.T., 1974. A method for preparating nematodes and other soft tissues for scanning electron microscopy. Mededelingen van den Rijksfaculteit Landbouwwetenschappen be Gent, 38: 1685-1695.

Fernández Rubio, F., 1985. Un nuevo modelo de trampa de luz portátil automática para caza de insectos. Boletín de la Estación Central de Ecología, 14(28): 91-102.

GARCÍA, R., 1998 Invertebrados artrópodos más representativos del P. N. de la Caldera de Taburiente. Págs. 188-191. In: A. Palomares (ed.). Guía de visita del P. $N$. de la Caldera de Taburiente. O. A. Parques Nacionales. Madrid. 205 pp.

García, R., Domingo T. \& SÁnchez A., 2001. Contribución al conocimiento de la fauna cavernícola del Bejenado (La Palma, Islas Canarias). Vulcania, 5: 39-49.

García, R., Govantes, F. \& Martín, M. A., 1997. Concepto de Espeleología Volcánica Canaria. Cabildo Insular de La Palma y Sociedad La Cosmológica. Santa Cruz de La Palma. 118 pp.

Hernández, A.J. \& PAstor, J., 1989. Técnicas analíticas para el estudio de las interacciones suelo-planta. Henares: Revista de Geología, 3: 67-102.

IZQUIERDO, I., MARTín, J. L., ZuRITA, N. \& ARACHAVALETA, M. (eds.), 2001. Lista de especies silvestres de Canarias (hongos, plantas y animales terrestres) 2001. Consejería de Política Territorial y Medio Ambiente, Gobierno de Canarias. Santa Cruz de Tenerife. 437 pp.

Koponen, M. \& Askew, R. R., 2002. Chalcids from Madeira, Canary Islands and Azores (Hymenoptera, Chalcidoidea). Vieraea, 30: 115-145.
Marcos-García, M. A., 1988. Métodos generales de captura. In: Barrientos, J. A. (Coord.). Bases para un curso práctico de Entomología. Asociación Española de Entomología. Salamanca: 11-24.

Martín-PierA, F., 2000. Introducción. In: F. Martín Piera, J. J. Morrone y A. Melic, A. (eds.). Hacia un Proyecto Cyted para el inventario y estimación de la diversidad entomológica en Iberoamérica: PrIBES 2000. m3m-Monografías Tercer Milenio, vol. 1. Sociedad Entomológica Aragonesa (SEA). Zaragoza: 19-31.

Monserrat, V. J., 2002. New data on the dusty wings from Africa and Europe (Insecta, Neuroptera, Coniopterygidae). Graellsia, 58(1): 3-19.

Nieves-Aldrey, J. L. \& Rey del Castillo, C., 1991. Ensayo preliminar sobre la captura de insectos por medio de una trampa "Malaise" en la sierra de Guadarrama (España), con especial referencia a los himenópteros (Insecta, Hymenoptera). Ecología, 5: 383-403.

Ortiz-SÁnchez, F. J. \& Aguirre-Segura, A., 1993. Efecto del color sobre las capturas de abejas mediante trampas 'Moericke' en el sur de España (Hymenoptera, Apoidea). Graellsia, 49: 63-71.

Ramazzotti, G. \& Maucci, W., 1983. Il Philum Tardigrada. III edizione riveduta e aggiornata. Memorie dell'Istituto Italiano di Idrobiologia, 41: 11012.

Ramsay, G. W., 1986. The taxonomic impediment to conservation. The Weta, 9(2): 60-62.

SAntos, A., 1983. Vegetación y flora de La Palma. Interinsular Canaria, S.A. Santa Cruz de Tenerife. $348 \mathrm{pp}$.

Southey, J. F. (ed.), 1978. Plant Nematology. Ministry of Agriculture, Fisheries and Food. London. 440 pp.

Townes, A., 1972. A light-weigth Malaise trap. Entomological News, 83: 239-247.

Valdecasas, A. G., 2002. Some water mites (Acari, Hydrachnidia) from Caldera de Taburiente Nacional Park (La Palma, Canary Islands). Graellsia, 58(2): 69-74.

Valdecasas, A. G. \& Baltanás, A., 1989. A note on the use of Angelier's fluid for freshwater invertebrates. Archiv für Hydrobiologie, 115: 313-316.

Wilson, E. O., (1985). The biological diversity crisis: A challenge to science. Issues in Science and Technology, 2: 20-29. 
Apéndice.- Inventario de novedades taxonómicas y faunísticas encontradas hasta el momento en el P.N. de La Caldera de Taburiente. $\mathrm{Pa} .=$ novedades para la fauna insular de $\mathrm{La} \mathrm{Palma} ; \mathrm{Ca} .=$ novedades para la fauna del archipiélago canario; $\mathrm{Ci} .=$ novedades para la Ciencia; $\mathrm{M}=$ Trampa Malaise; $\mathrm{YT}=$ Trampa de Moericke; $\mathrm{TC}=$ Pitfall; $\mathrm{TS}=$ Trampa del MSS; $\mathrm{C}=\mathrm{Pitfall}$ en cuevas; $\mathrm{V}=$ Mangueo de vegetación; $\mathrm{B}=$ Muestras Winkler-Berlese; $\mathrm{N}=$ Muestras para Nematoda; $\mathrm{A}=$ Muestreo de fauna dulceacuícola; $\mathrm{X}=$ Xilófagos; $\mathrm{F}=$ Necrófagos; $\mathrm{L}=$ Trampa de luz; $\mathrm{P}=$ Fauna epigea; $\mathrm{T}=$ Muestras para Tardigrada; $\mathrm{W}=$ Winkler; $\mathrm{E}=\mathrm{Muestreos}$ nocturnos. $\bigcirc=$ género y especie nuevos; $\mathrm{O}=$ sólo especie nueva; ?= novedad sin confirmar.

Appendix.- Inventory of taxonomic and faunistic novelties found for the moment in the N. P. of La Caldera de Taburiente. Pa.= novelties for the fauna of La Palma I.; Ca.= novelties for the fauna of the Canary Is.; Ci.= new taxa for Science; $\mathrm{M}=\mathrm{Malaise}$ trap; $\mathrm{YT}=$ Moericke trap; $\mathrm{TC}=$ pitfall trap; $\mathrm{TS}=\mathrm{MSS}$ trap; $\mathrm{C}=$ pitfall in caves; $\mathrm{V}=$ plant netting; $\mathrm{B}=$ Winkler-Berlese sampling; $\mathrm{N}=$ Nematoda sampling; $A=$ freshwater fauna sampling; $X=$ xylophagous fauna sampling; $F=$ necrophagous fauna sampling; $L=$ light trap; $\mathrm{P}=$ epigean fauna sampling; $\mathrm{T}=$ sampling for Tardigrada; $\mathrm{W}=$ Winkler samples; $\mathrm{E}=$ nocturnal sampling. $\bigcirc=$ new genus and species; $\bigcirc=$ only new species; ?= unconfirmed novelty.

\begin{tabular}{|c|c|c|}
\hline Grupo taxonómico & $\mathbf{P a}$ & Localidades \\
\hline \multicolumn{3}{|c|}{$\begin{array}{l}\text { Phylum Platyhelminthes } \\
\text { Clase Turbellaria }\end{array}$} \\
\hline Orden Tricladida & $\mathrm{O}$ & \\
\hline Dugesiidae & $\mathrm{O}$ & \\
\hline Dugesia sp. & - & $\begin{array}{l}\text { A1-VII; A1-X; A2-I; A2-III; A2-X; A6-VII; A20-IX; } \\
\text { A27-I; A27-VII; A29-VII; A34-I; A34-VII; A34-IX; } \\
\text { A34-X; A35-IX; A36-X; A37-VII; A37-X; A38-II; } \\
\text { A38-VII; A38-IX; A38-X; A39-VII; A39-X; A44-X; } \\
\text { A59-I; A59-VII; A59-X }\end{array}$ \\
\hline
\end{tabular}

\section{Phylum Nemathelminthes \\ Clase Nematoda}

\begin{tabular}{|c|c|c|c|}
\hline $\begin{array}{l}\text { Orden Araeolaimida } \\
\text { Plectidae }\end{array}$ & & & \\
\hline Plectus sp. & - & - & N62-VII; N70-VII \\
\hline \multicolumn{4}{|l|}{ Prismatolaimidae } \\
\hline Prismatolaimus sp. & - & - & N29-VII \\
\hline \multicolumn{4}{|l|}{$\begin{array}{l}\text { Orden Enoplida } \\
\text { Alaimidae }\end{array}$} \\
\hline Alaimus sp. & - & - & N64-VII \\
\hline \multicolumn{4}{|l|}{$\begin{array}{l}\text { Orden Dorylaimida } \\
\text { Aporcelaimidae }\end{array}$} \\
\hline Aporcelaimus sp. & - & - & N6-VIII; N63-VII \\
\hline \multicolumn{4}{|l|}{ Dorylaimidae } \\
\hline Dorylaimus sp. & - & - & N42-VII; N59-VII; N63-VII \\
\hline Eudorylaimus sp. & - & - & N6-VIII \\
\hline Mesodorylaimus sp. & - & - & N42-VII \\
\hline \multicolumn{4}{|l|}{ Longidoridae } \\
\hline Longidorus sp. & $\bullet$ & - & N63-VII \\
\hline Xiphinema sp. & $\bullet$ & - & N49-VII; N63-VII; N64-VII \\
\hline
\end{tabular}


Orden Mononchida

Cephalobidae

\begin{tabular}{|c|c|c|c|}
\hline Acrobeles sp. & $\bullet$ & $\bullet$ & $\begin{array}{l}\text { N6-VIII; N10-VII; N42-VII; N59-VII; N62-VII; } \\
\text { N63-VII; N70-VII }\end{array}$ \\
\hline Acrobeloides sp. & • & - & N59-VII; N62-VII \\
\hline Cephalobus sp. & - & - & N29-VII; N45-VII; N49-VII; N62-VII \\
\hline \multicolumn{4}{|l|}{ Mononchidae } \\
\hline Coomansus sp. & - & - & N10-VII; N59-VII; N62-VII; N63-VII \\
\hline Mononchus sp. & - & - & N42-VII \\
\hline \multicolumn{4}{|c|}{ Orden Tylenchida } \\
\hline Aphelenchus sp. & - & - & N45-VII; N59-VII; N62-VII; N63-VII \\
\hline \multicolumn{4}{|c|}{ Criconematidae } \\
\hline Criconemoides sp. & - & - & N59-VII; N62-VII \\
\hline \multicolumn{4}{|c|}{ Paratylenchidae } \\
\hline Paratylenchus sp. & - & • & $\begin{array}{l}\text { N6-VIII; N29-VII; N49-VII; N59-VII; N62-VII; } \\
\text { N63-VII; N64-VII }\end{array}$ \\
\hline \multicolumn{4}{|l|}{ Tylenchidae } \\
\hline Filenchus sp. & $\bullet$ & $\bullet$ & $\begin{array}{l}\text { N6-VIII; N10-VII; N49-VII; N59-VII; N63-VII; } \\
\text { N64-VII }\end{array}$ \\
\hline Lelenchus sp. & - & $\bullet$ & N33-VII \\
\hline Tylenchus sp. & $\bullet$ & $\bullet$ & $\begin{array}{l}\text { N6-VIII; N33-VII; N45-VII; N49-VII; N59-VII; } \\
\text { N62-VII; N63-VII }\end{array}$ \\
\hline \multicolumn{4}{|c|}{$\begin{array}{l}\text { Orden Rhabditida } \\
\text { Rhabditidae }\end{array}$} \\
\hline Rhabditis sp. & - & - & N29-VII; N33-VII; N45-VII; N62-VII; N63-VII \\
\hline
\end{tabular}

Phylum Annelida

Clase Oligochaeta

Orden Haplotaxida

\begin{tabular}{l|l|l|l|l}
\hline \multicolumn{1}{|c|}{ Acanthodrilidae } & $\bigcirc$ & & & \\
\hline Microscolex sp. & $\bullet$ & & & P34-IX \\
\hline
\end{tabular}

\section{Phylum Tardigrada}

Clase Heterotardigrada

\begin{tabular}{l|r|r|r|r}
\hline \multicolumn{1}{c|}{ Orden Echiniscoidea } & O & & & \\
\hline \multicolumn{1}{c|}{ Echiniscidae } & O & & & \\
\hline Echiniscus canadensis Murray, 1910 & $\bullet$ & O & & T47-III \\
\hline Echiniscus mediantus Marcus, 1930 & $\bullet$ & $\bigcirc$ & & T47-III \\
\hline Echiniscus trisetosus Cuènot, 1932 & $\bullet$ & $\bigcirc$ & & T47-III \\
\hline
\end{tabular}

\section{Clase Eutardigrada}

\begin{tabular}{l|r|r|r|r}
\hline \multicolumn{1}{c|}{ Orden Parachela } & O & & & \\
\hline \multicolumn{1}{l|}{ Macrobiotidae } & O & & & \\
\hline Macrobiotus gr. hufelandi Schultze, 1834 & $\bullet$ & & & T47-III \\
\hline Macrobiotus occidentalis Murray, 1910 & $\bullet$ & O & & T47-III \\
\hline Minibiotus furcatus (Ehrenberg, 1859) & $\bullet$ & ○ & & T47-III \\
\hline
\end{tabular}




\begin{tabular}{l|r|r|r|r}
\hline Minibiotus n sp. & ○ & ○ & ○ & T47-III \\
\hline Orden Apochela & & & & \\
\hline Milnesiidae & $\bigcirc$ & & & \\
\hline Milnesium tardigradum Doyére, 1840 & $\bigcirc$ & & & \\
\hline
\end{tabular}

\section{Phylum Arthropoda \\ Clase Arachnida}

Orden Pseudoscorpiones

Chtoniidae

Paraliochthonius mirus Mahnert, 2002

Olpiidae

Calocheirus canariensis (Beier, 1965)

Orden Opiliones

Phalangiidae

Bunochelis sp. $n$.

Bunochelis spinifera (Lucas, 1839) cf.

\section{Orden Acari}

Amblyseidae

Amblyseius cucumeris (Oudemans, 1930)

Amblyseius messor Wainstein, 1960

\begin{tabular}{|c|c|c|c|c|}
\hline Ambiyserus messor wainstein, 1900 & 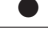 & ( & & $\mathrm{B} / 4-\mathrm{XII}$ \\
\hline Anystidae & $\mathrm{O}$ & $O$ & & \\
\hline Anystis sp. & $\bullet$ & - & & M59-XII \\
\hline Tarsotomus sp. & $\bullet$ & - & & TC49-X \\
\hline Ascidae & $\mathrm{O}$ & $\mathrm{O}$ & & \\
\hline Proctolaelaps pygmaeus (Muller, 1860) & - & - & & B74-XII; B77-IX \\
\hline Aturidae & O & $\mathrm{O}$ & & \\
\hline Aturus atlanticus Lundblad, 1942 & - & - & & A39-III; A44-III \\
\hline Bdellidae & O & O & & \\
\hline Bdellodes (Hoploscirus) sp. 1 & - & - & $?$ & TC49-XII; B77-IX \\
\hline Cyta latirostris (Hermann, 1804) & - & - & & YT59-IX; B63-XII; B77-IX \\
\hline Odontoscirus sp. & - & - & & P63-III \\
\hline Spinibdella tenuirostris Ewing, 1975 & - & - & & B6-XI; B63-XII \\
\hline Bryobiidae & $\mathrm{O}$ & & & \\
\hline Bryobia sp. & - & & & B74-XII \\
\hline Caligonellidae & O & $\mathrm{O}$ & & \\
\hline Coptocheles sp. & - & $\mathbf{0}$ & & B74-XII \\
\hline Camisiidae & O & & & \\
\hline Camisia borealis (Thorell, 1871) & - & $\mathrm{O}$ & & B63-XII \\
\hline Camisia spinifer (Koch, 1835) & - & & & B63-XII \\
\hline Carabodidae & O & & & \\
\hline Odontocepheus elongatus (Michael, 1879) & - & & & B63-XII; B74-XII \\
\hline Ceratoppiidae & $\mathrm{O}$ & & & \\
\hline Ceratoppia bipilis (Hermann, 1804) & - & & & YT63-IX; B63-XII \\
\hline Ceratozetidae & $\mathrm{O}$ & & & \\
\hline Trichoribates brevicuspis Mihelcic, 1958 & O & O & & YT63-IX \\
\hline Trichoribates brevicuspis Mihelcic, $1958 \mathrm{cf}$. & $\mathrm{O}$ & $\mathrm{O}$ & $?$ & YT63-VIII; YT63-IX \\
\hline
\end{tabular}




\begin{tabular}{|c|c|c|c|c|}
\hline Chamobatidae & $\mathrm{O}$ & & & \\
\hline Chamobates pusillus (Berlese, 1895) & - & & & B6-XI; B74-XII \\
\hline Cosmochthoniidae & $\mathrm{O}$ & & & \\
\hline Cosmochthonius sp. & - & & & B74-XII \\
\hline Cryptognathidae & $\mathrm{O}$ & O & & \\
\hline Cryptognathus corrugis Summers \& Chaudri, 1965 cf. & - & - & $?$ & B74-XII \\
\hline Cunaxidae & $\mathrm{O}$ & $\mathrm{O}$ & & \\
\hline Cunaxa setirostris (Hermann, 1804) & - & - & & B74-XII \\
\hline Cunaxoides biscutum (Nesbitt, 1936) cf. & - & - & $?$ & B63-XII \\
\hline Cunaxoides longistriatus Kuznetsov \& Livshitz, 1975 cf. & - & - & $?$ & B77-IX \\
\hline Cymbaeremaeidae & $\mathrm{O}$ & & & \\
\hline Cymbaeremaeus cymba (Nicolet, 1855) & - & & & YT63-IX \\
\hline Scapheremaeus patella (Berlese, 1886) & - & & & YT6-VIII; YT63-VIII; YT63-IX \\
\hline Damaeidae & $\mathrm{O}$ & & & \\
\hline Damaeus recasensis Capilla, 1971 & $\bullet$ & & & B74-XII \\
\hline Erythraeidae & $\mathrm{O}$ & $\mathrm{O}$ & & \\
\hline Abrolophus sp. & - & - & & M59-XII \\
\hline Erythraeus sp. & - & - & & TC49-X \\
\hline Eupodidae & $\mathrm{O}$ & O & & \\
\hline Cocceupodes sp. & - & - & & B6-XI; B63-XII \\
\hline Eupodes berlesei Sig Thor, 1891 cf. & - & - & $?$ & B63-XII; B74-XII \\
\hline Eupodes ereneytoides Strandtmann \& Prasse, 1977 & - & - & & B77-IX \\
\hline Eupodes longisetatus Strandtmann, 1964 cf. & - & - & $?$ & B63-XII \\
\hline Eupodes ocellatus Willmann, 1952 cf. & $\bullet$ & - & $?$ & B63-XII \\
\hline Feltriidae & $\mathrm{O}$ & $\mathrm{O}$ & & \\
\hline Feltria menzeli Walter, 1922 & - & - & & A39-III; A40-III \\
\hline Galumnatidae & $O$ & & & \\
\hline Galumna alatum (Hermann, 1804) & - & & & B6-XI \\
\hline Hemileiidae & O & & & \\
\hline Hemileius humeralis Pérez-Iñigo, 1991 & - & $\mathrm{O}$ & & B6-XI \\
\hline Hermanniellidae & O & & & \\
\hline Hermanniella reticulata Sitnikova, 1974 & - & $\mathrm{O}$ & & B74-XII \\
\hline Histiostomatidae & $\mathrm{O}$ & $O$ & & \\
\hline Histiostoma sp. & - & - & & B74-XII \\
\hline \multicolumn{5}{|l|}{ Hydryphantidae } \\
\hline Gen. nov & - & - & - & A34-III \\
\hline Protzia lata Walter, 1906 cf. & - & - & $?$ & $\begin{array}{l}\text { A34-I; A34-III; A34-X; A39-X; A40-III; A44-III; } \\
\text { A44-X; A59-III }\end{array}$ \\
\hline Hygrobatidae & $\mathrm{O}$ & & & \\
\hline Atractides gomerae Lundblad, 1962 & - & & & A27-I; A34-IX; A38-VII; A40-III; A44-X; A59-X \\
\hline Laelapidae & $\mathrm{O}$ & & & \\
\hline Cosmolaelaps claviger (Berlese, 1883) & - & - & & B74-XII \\
\hline Geolaelaps sp. & - & - & & B74-XII \\
\hline Lebertiidae & $\mathrm{O}$ & & & \\
\hline Lebertia fimbriata (Thor, 1899) & - & & & A34-I; A39-III; A40-III; A44-III \\
\hline Limnesiidae & $\mathrm{O}$ & & & \\
\hline Limnesia martianezi Lundblad, 1962 & - & & & A36-X \\
\hline Macrochelidae & $\mathrm{O}$ & $\mathrm{O}$ & & \\
\hline Macrocheles n.sp. ? & - & - & $?$ & B77-IX \\
\hline Macrocheles scutatiformis Petrova, 1967 & - & - & & B74-XII \\
\hline Pseudoparasitus dentatus (Halbert, 1920) & - & - & & B6-XI; B74-XII \\
\hline
\end{tabular}




\begin{tabular}{|c|c|c|c|c|}
\hline Micreremidae & $\mathrm{O}$ & & & \\
\hline Micreremus sp. & - & & & YT6-VIII \\
\hline Nanorchestidae & O & O & & \\
\hline Nanorchestes pulvinar Grandjean, 1942 & - & - & & B74-XII \\
\hline Oppiidae & $\mathrm{O}$ & & & \\
\hline Ramusella (Ramusella) sp. & - & & & B77-IX \\
\hline Ramusella (Rectoppia) sp. & - & & & B63-XII; B74-XII \\
\hline Oribatellidae & $\mathrm{O}$ & & & \\
\hline Oribatella berlesei (Michael, 1898) & - & $\mathrm{O}$ & & B77-IX \\
\hline Oribatulidae & $\mathrm{O}$ & & & \\
\hline Phauloppia lucorum (Koch, 1841) & - & & & YT63-VIII; YT63-IX \\
\hline Zygoribatula frisiae (Oudemans, 1916) & - & & & B63-XII; B74-XII \\
\hline Zygoribatula undulata Berlese, 1917 cf. & - & $\mathrm{O}$ & $?$ & B16-XI; TC49-IX; TC49-X \\
\hline Parasitidae & $\mathrm{O}$ & $\mathrm{O}$ & & \\
\hline Parasitus americanus Berlese, 1905 cf. & - & - & $?$ & B74-XII \\
\hline Poecilochirus sp. & - & - & & B74-XII \\
\hline Penthaleidae & $O$ & $\mathrm{O}$ & & \\
\hline Penthaleus major (Dugès, 1834) & - & - & & B63-XII \\
\hline Penthalodidae & $\mathrm{O}$ & $\mathrm{O}$ & & \\
\hline Penthalodes boneti Baker, 1946 cf. & - & - & $?$ & B63-XII \\
\hline Phenopelopidae & $\mathrm{O}$ & $\mathrm{O}$ & & \\
\hline Eupelops hirtus (Berlesse, 1916) & - & & & YT63-VIII; YT63-IX; B63-XII \\
\hline Phthiracaridae & $\mathrm{O}$ & & & \\
\hline Phthiracarus piger (Scopoli, 1763) cf. & - & - & $?$ & B74-XII \\
\hline Rhagidiidae & $\mathrm{O}$ & $\mathrm{O}$ & & \\
\hline Foveocheles sp. & - & - & & B63-XII \\
\hline Robustocheles tricuspidata Zacharda, 1980 cf. & $\mathbf{0}$ & $\mathbf{0}$ & $?$ & B63-XII \\
\hline Robustocheles tricuspidata Zacharda, 1980 & - & - & & P63-III \\
\hline Rhodacaridae & $\mathrm{O}$ & $\mathrm{O}$ & & \\
\hline Protogamasellus primitivus Karg, 1962 & - & - & & B77-IX \\
\hline Smarididae & $\mathrm{O}$ & $\mathrm{O}$ & & \\
\hline Smaris sp. & - & - & & TC49-X \\
\hline Steganacaridae & $\mathrm{O}$ & & & \\
\hline Steganacarus hirsutus Pérez-Iñigo, 1974 & - & & & B6-XI; B63-XII; B74-XII \\
\hline Stigmaeidae & $\mathrm{O}$ & $\mathrm{O}$ & & \\
\hline Ledermuellenopsis plumosa Willmann, 1951 cf. & - & - & $?$ & B63-XII \\
\hline Suctobelbidae & $\mathrm{O}$ & & & \\
\hline Suctobelbella subcornigera (Forsslund, 1941) & - & - & & B74-XII \\
\hline Tarsonemidae & $\mathrm{O}$ & & & \\
\hline Tarsonemus sp. & - & - & & B63-XII \\
\hline Tectocepheidae & $\mathrm{O}$ & & & \\
\hline Tectocepheus sarekensis Trägârdh, 1910 & - & & & B6-XI \\
\hline \multicolumn{5}{|l|}{ Torrenticolidae } \\
\hline Torrenticola gomerae Lundblad, 1972 & $\mathrm{O}$ & & & A2-I; A27-I; A38-VII; A39-III; A40-III \\
\hline Tydeidae & $\mathrm{O}$ & $\mathrm{O}$ & & \\
\hline Pseudotrioptydeus sp. & - & - & & B74-XII \\
\hline Tydeus placitus Livshitz, 1973 cf. & - & - & $?$ & B77-IX \\
\hline Uropodidae & $\mathrm{O}$ & $\mathrm{O}$ & & \\
\hline Olodiscus minimus C.L.Koch, 1841 & - & - & & B6-XI; B74-XII \\
\hline Veigaiidae & $\mathrm{O}$ & $\mathrm{O}$ & & \\
\hline Veigaia planicola Berlese, 1892 & - & $\mathbf{0}$ & & B6-XI; V43-VII \\
\hline
\end{tabular}




\begin{tabular}{|c|c|c|c|c|}
\hline Xenillidae & $\mathrm{O}$ & & & \\
\hline Xenillus tegeocranus (Hermann, 1804) & 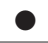 & & & B74-XII \\
\hline Zerconidae & $\mathrm{O}$ & 0 & & \\
\hline Zercon n.sp. & $\bullet$ & $\bullet$ & $\mathrm{O}$ & B63-XII \\
\hline \multicolumn{5}{|l|}{ Orden Araneae } \\
\hline Mangora acalypha (Walckenaer, 1802) & - & & & V51-I; P59-III; V59-VII \\
\hline Zygiella x-notata (Clerck, 1758) cf. & $\mathrm{O}$ & $O$ & $?$ & P24-I \\
\hline Filistatidae & $\mathrm{O}$ & & & \\
\hline Filistata insidiatrix (Forsskål, 1775) & - & ○ & & M67-VIII \\
\hline Scytodidae & $\mathrm{O}$ & & & \\
\hline Scytodes bertheloti Lucas, 1838 & - & & & TC49-X \\
\hline
\end{tabular}

Clase Ostracoda

Orden Podocopida

Cyprididae

\begin{tabular}{|l|l|l|l|l}
\hline Herpetocypris brevicaudata Kaufmann, 1900 & O & & & A27-I \\
\hline
\end{tabular}

\section{Clase Malacostraca}

\section{Orden Isopoda}

Armadillidiidae

Armadillidium album Dollfus, $1887 \mathrm{cf}$

Armadillidium sp. 1

Armadillidium sp. 2

\begin{tabular}{|c|c|c|c|c|}
\hline & $?$ & $?$ & TC63-V \\
\hline$?$ & $?$ & $?$ & P34-IX \\
\hline$?$ & $?$ & $?$ & A34-III
\end{tabular}

Clase Diplopoda

Orden Polyxenida

Polyxenidae

\begin{tabular}{l|l|l|l|r|}
\hline Polyxenus fasciculatus Say, 1821 & O & & & B6-XI; TC63-VIII; B74-XII \\
\hline
\end{tabular}

Orden Julida

Julidae

Dolichoiulus n.sp?

\begin{tabular}{l|l|l} 
? & $?$ & $?$
\end{tabular}

C22-IV; C22-X; C22-XI; C22-XII

\section{Clase Chilopoda}

\section{Orden Lithobiomorpha}

Henicopidae

\begin{tabular}{|c|c|c|c|c|}
\hline Lamyctes coeculus Brölemann, 1889 & O & & & P59-III \\
\hline Lamyctes mauresi Demange, 1981 & O & & & P73-III \\
\hline \multicolumn{5}{|l|}{ Lithobiidae } \\
\hline Lithobius n.sp. & O & $\mathrm{O}$ & $\mathrm{O}$ & C22-VI; C22-X; C22-XII; C25-VI \\
\hline \multicolumn{5}{|l|}{ Orden Scolopendromorpha } \\
\hline Scolopendridae & O & & & \\
\hline Scolopendra valida Lucas, 1840 & $\bullet$ & & & P37-VI; P32-V \\
\hline
\end{tabular}




\begin{tabular}{l|r|r|r|c}
\hline \multicolumn{1}{c|}{ Orden Geophilomorpha } & & & & \\
\hline Dignathodontidae & O & & & \\
\hline Henia bicarinata (Meinert, 1870) & $\bullet$ & & & B63-XII \\
\hline Schendylidae & O & & & \\
\hline Schendyla peyerimhoffi Brölemann, 1911 & $\bullet$ & $\bullet$ & & B63-XII; B74-XII \\
\hline
\end{tabular}

\section{Clase Collembola}

\section{Orden Poduromorpha}

Hypogastruridae

\begin{tabular}{|c|c|c|c|c|}
\hline Xenylla maritima Tüllberg, 1869 & O & & & TC63-V; B63-XII \\
\hline Neanuridae & $\mathrm{O}$ & & & \\
\hline Brachystomella curvula Gisin, 1948 & 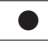 & & & B16-XI \\
\hline Brachystomella parvula (Schäffer, 1896) & $\bullet$ & O & & B77-IX \\
\hline Pratanurida boerneri (Schött, 1902) & ○ & $\bullet$ & & B74-XII \\
\hline Onychiuridae & 0 & & & \\
\hline Mesaphorura orousseti Najt, Thibaud y Weiner, 1990 & ○ & O & & B74-XII \\
\hline Metaphorura denisi Simón, 1986 & - & - & & B6-XI \\
\hline \multicolumn{5}{|l|}{$\begin{array}{l}\text { Orden Entomobryomorpha } \\
\text { Entomobryidae }\end{array}$} \\
\hline Entomobrya atrocincta Schött, 1897 & $\bigcirc$ & O & & $\begin{array}{l}\text { A2-X; M6-I; M6-II; M6-XI; V44-IX; TC49-X; M59- } \\
\text { I; M59-XII; V77-V; B77-IX }\end{array}$ \\
\hline Entomobrya n. sp. pr. multifasciata (Tullberg, 1871) & 0 & O & 0 & M6-IX; YT6-IX; TC59-III; B63-XII \\
\hline Entomobrya n. sp. & O & O & $\mathrm{O}$ & $\begin{array}{l}\text { YT6-IX; B6-XI; TC49-XII; M59-XII; YT63-IX; } \\
\text { B74-XII; B77-IX }\end{array}$ \\
\hline Entomobrya sp. 1 & ? & ? & $?$ & $\begin{array}{l}\text { M6-I; M6-II; M6-XI; M6-XII; W15-III; P26-I; P32- } \\
\text { V; A34-I; A34-III; A38-VII; P44-III; M49-XI; M49- } \\
\text { XII; M50-II; V51-I; W61-III; M63-I; M63-II; M63- } \\
\text { XII; P67-III; V67-III; TC49-I; TC50-IV; TC50-II; } \\
\text { TC50-III; TC63-II; TC63-IV; TC63-VII; TC67-X }\end{array}$ \\
\hline Entomobrya sp. 2 & $?$ & $?$ & $?$ & $\begin{array}{l}\text { TC6-III; TC6-V; W15-III; A27-I; V27-I; V44-IX; } \\
\text { M49-XII; M49-XI; TC50-II; TC50-IV; V54-V; M59- } \\
\text { I; TC59-V; TC59-IX; E59-IX; TC63-I; TC63-II; } \\
\text { TC63-III; TC63-V; TC63-VI; TC63-VIII; M63-XI; } \\
\text { TC67-IX; V68-V; V71-III; V77-V }\end{array}$ \\
\hline Entomobrya sp. 3 & $?$ & ? & $?$ & P38-II \\
\hline Heteromurus nitidus margaritaria Wankel, 1860 & $?$ & $?$ & & C22-VI; C22-XI \\
\hline Lepidocyrtus nigrescens Szeptcyky, 1967 & ○ & $\bigcirc$ & & $\begin{array}{l}\text { M6-I; M6-II; M6-IX; M6-XI; M6-XII; TC6-II; TC6- } \\
\text { III; TC6-IV; TC6-V; TC6-VI; TC6-VIII; P6-III; } \\
\text { YT6-IX; D7-V; P8-V; P26-I; P44-III; P46-V; M59-I; } \\
\text { E59-III; W59-V; TC59-I; TC59-II; TC59-III; TC59- } \\
\text { V; TC59-VI; P72-III }\end{array}$ \\
\hline \multicolumn{5}{|l|}{ Isotomidae } \\
\hline Cryptopygus bituberculatus Wahlgren, $1906 \mathrm{cf}$. & - & $\bullet$ & $?$ & B6-XI \\
\hline Cryptopygus debilis Cassagnau, 1959 & - & $\bullet$ & & B63-XII \\
\hline Cryptopygus thermophilus (Axelson, 1900) & - & $\bullet$ & & TC6-I \\
\hline Folsomides zairensis Martynova, 1978 cf. & O & ? & $?$ & B63-XII \\
\hline Isotomurus gr. palustris (Müller, 1776) sp. 1 & ○ & $?$ & $?$ & P44-X; B74-XII \\
\hline Isotomurus gr. palustris (Müller, 1776) sp. 2 & - & $?$ & $?$ & $\begin{array}{l}\text { P2-I; A34-III; A34-IX; A34-X; A36-X; A37-III; } \\
\text { A38-VII; A39-X; M59-I; W61-III }\end{array}$ \\
\hline
\end{tabular}




\begin{tabular}{|c|c|c|c|c|}
\hline Parisotoma notabilis (Schäffer, 1896) & - & & & B63-XII; B74-XII; B77-IX \\
\hline Vertagopus sp. & - & $\bullet$ & & A3-VII; M6-I; M6-XII; P38-II; A39-X; V53-I \\
\hline \multicolumn{5}{|l|}{ Orden Symphypleona } \\
\hline Arrhopalitidae & $\mathrm{O}$ & & & \\
\hline Arrhopalites n. sp. & O & $\mathrm{O}$ & O & $\mathrm{C} 22-\mathrm{XI}$ \\
\hline Bourletiellidae & $\mathrm{O}$ & & & \\
\hline Fasciosminthurus canariensis (Paclt, 1964) cf. & ○ & $?$ & $?$ & M6-VIII; YT6-VIII; YT63-VIII \\
\hline Dicyrtomidae & $\mathrm{O}$ & $\mathrm{O}$ & & M6-I; M6-XII; P38-II; A39-X; V53-I \\
\hline \multicolumn{5}{|l|}{ Katiannidae } \\
\hline Sminthurinus n.sp. & $\mathrm{O}$ & $\mathrm{O}$ & $\mathrm{O}$ & M49-XI; B63-XII; V71-II; V73-V; V74-III \\
\hline Stenognathellus gr. polygonalis Hüher, 1967 & - & - & & TC49-X \\
\hline \multicolumn{5}{|l|}{ Sminthuridae } \\
\hline Caprainea sp. & - & $\bullet$ & & B63-XII \\
\hline Sminthurus n. sp. & $\bullet$ & $\bullet$ & O & $\begin{array}{l}\text { A37-I; V41-III; M49-XI; E59-III; W61-III; P72-III; } \\
\text { V74-II }\end{array}$ \\
\hline Sminthurididae & $\mathrm{O}$ & & & \\
\hline Sphaeridia sp. & - & - & & M6-XII; TC63-I; B63-XII \\
\hline
\end{tabular}

Clase Insecta

Orden Blattaria

Ectobiidae

\begin{tabular}{|c|c|c|c|c|}
\hline Phyllodromica n. sp. & $O$ & $\mathrm{O}$ & $O$ & P13-III \\
\hline \multicolumn{5}{|l|}{ Orden Psocoptera } \\
\hline Amphientomidae & $\mathrm{O}$ & & & \\
\hline Nephax fortunatus (Navás, 1917) & $\mathbf{0}$ & & & TC59-IX; TC67-VIII; TC67-X \\
\hline \multicolumn{5}{|l|}{ Ectopsocidae } \\
\hline Ectopsocus meridionalis Ribaga, 1904 & $\mathrm{O}$ & $\mathrm{O}$ & & M6-II \\
\hline Ectopsocus vachoni Badonnel, 1945 & O & & & $\begin{array}{l}\text { M6-II; M6-VII; M6-VIII; M6-IX; M6-X; M6-XI; M6- } \\
\text { XII; B16-XI; V36-IX; V41-I; P44-IX; M49-I; M49- } \\
\text { XI; M49-XII; TC49-I; TC49-X; TC50-II; TC50-III; } \\
\text { M50-II; M50-IV; M59-XII; YT63-VIII; YT63-IX; } \\
\text { M63-II; M63-VII; M63-VIII; M63-XI; M63-XII }\end{array}$ \\
\hline \multicolumn{5}{|l|}{ Elipsocidae } \\
\hline Elipsocus lanciloticus Baz, 1991 & 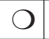 & & & M63-I; M63-II; M63-IV; M63-XI; M63-XII \\
\hline \multicolumn{5}{|l|}{ Lachesillidae } \\
\hline Lachesilla meinanderi Liendhard, 1998 & $\mathrm{O}$ & & & V6-III \\
\hline \multicolumn{5}{|l|}{ Liposcelididae } \\
\hline Liposcelis paetula Broadhead, 1950 & $\mathrm{O}$ & & & B74-XII \\
\hline Pachytroctidae & $O$ & & & \\
\hline Nymphotroctes denisi Badonnel, 1931 & ○ & & & M50-VII \\
\hline \multicolumn{5}{|l|}{ Psocidae } \\
\hline Atlantopsocus semicircularis Baz, 1989 & $O$ & & & M6-XI \\
\hline Psyllipsocidae & $O$ & & & \\
\hline Psyllipsocus ramburii Selys-Longchamps, 1872 & ○ & & & C22-VI; C23-IV; C23-VI; C25-IX \\
\hline \multicolumn{5}{|l|}{ Trogiidae } \\
\hline Lepinotus reticulatus Enderlein, 1905 & ○ & & & $\begin{array}{l}\text { YT6-VIII; M6-IX; TS8-VIII; M59-XI; M63-VII; } \\
\text { YT63-IX; B63-XII; B74-XII }\end{array}$ \\
\hline
\end{tabular}




\begin{tabular}{|c|c|c|c|c|}
\hline $\begin{array}{l}\text { Orden Thysanoptera } \\
\text { Aelothripidae }\end{array}$ & & & & \\
\hline Aelothrips intermedius Bagnall, 1934 cf. & $\mathrm{O}$ & $O$ & $?$ & M6-II; V31-III; V31-V; YT63-VIII; V67-VI; V71-II \\
\hline \multicolumn{5}{|l|}{ Thripidae } \\
\hline Chirothrips manicautus (Haliday, 1836) & $\mathrm{O}$ & $O$ & & V31-VI \\
\hline Scolothrips latipennis Priesner, $1950 \mathrm{cf}$. & - & $?$ & $?$ & M6-VII; M6-VIII; YT6-VIII; YT63-VIII \\
\hline \multicolumn{5}{|l|}{ Orden Hemiptera } \\
\hline Acanthosomatidae & $O$ & & & \\
\hline Cyphostethus tristriatus (Fabricius, 1787) & - & & & $\mathrm{V} 5-\mathrm{V}$ \\
\hline \multicolumn{5}{|l|}{ Aphididae } \\
\hline Acyrthosiphon pisum (Harris, 1776) & - & & & $\begin{array}{l}\text { V6-III; V31-III; V37-III; M50-IV; TC50-VI; M59- } \\
\text { IV; M63-IV; TC63-VI; V77-V }\end{array}$ \\
\hline Aploneura lentisici (Passerini, 1856) & - & & & M63-IV \\
\hline Aulacorthum solani (Kaltenbach, 1843) & - & & & YT6-VIII \\
\hline Cavariella aegopodii (Scopoli, 1763) & - & & & M6-V \\
\hline Coloradoa sp. & - & - & & M6-IX \\
\hline Eucarazzia elegans (Ferrari, 1872) & - & & & M6-II \\
\hline Eulachnus tauricus Bonzhko, 1961 & $\mathrm{O}$ & $O$ & & M6-VIII; YT63-VIII \\
\hline Hyalopterus pruni (Geoffroy, 1762) cf. & - & & & YT59-IX \\
\hline Lipaphis erysimi (Kaltenbach, 1843) & - & & & YT59-IX \\
\hline Neotoxoptera violae (Pergande, 1900) & - & $\bullet$ & & M6-V \\
\hline Schizaphis graminum (Rondani, 1852) & - & & & M6-V \\
\hline Sitobion fragariae (Walker, 1848) & $O$ & & & $\begin{array}{l}\text { M6-I; M6-V; M6-VII; V6-III; V6-V; V30-V; V54-V; } \\
\text { M59-I; E59-III; V71-V; V73-V; V76-VI }\end{array}$ \\
\hline Tetraneura nigriabdominalis (Sasaki, 1899) & - & - & & YT6-VIII \\
\hline Therioaphis trifolii (Monell, 1882) & - & & & M6-V; M50-IV; M63-IV; V73-V \\
\hline Uroleucon inulae (Ferrari, 1872) & $\mathrm{O}$ & $\mathrm{O}$ & & YT6-VIII; YT6-IX; YT59-IX \\
\hline Wahlgreniella nervata (Gillette, 1908) & - & & & $\begin{array}{l}\text { M6-VII; M6-VIII; YT6-VIII; M50-VII; M63-VII; } \\
\text { M63-VIII; YT63-VIII }\end{array}$ \\
\hline Hebridae & $O$ & & & \\
\hline $\begin{array}{l}\text { Merragata hebroides White, } 1877 \\
\quad \text { Lygaeidae } \\
\end{array}$ & - & & & A2-X; A12-IX; ?37-IX \\
\hline Emblethis verbasci (Fabricius, 1803) cf. & $\mathrm{O}$ & $?$ & $?$ & ?71-VIII \\
\hline Lamprodema maurum (Fabricius, 1803) & - & & & V17-VIII; ?71-VIII \\
\hline Megalonotus sabulicola (Thomson, 1870) & $O$ & & & B6-V \\
\hline Mesoveliidae & $\mathrm{O}$ & & & \\
\hline Mesovelia vittigera Horváth, 1895 & - & & & P59-IX \\
\hline \multicolumn{5}{|l|}{ Pseudococcidae } \\
\hline Phenacoccus aceris (Signolet, 1875) & - & $\mathrm{O}$ & & V14-VII \\
\hline \multicolumn{5}{|l|}{ Psyllidae } \\
\hline Acizzia acaciaebaileyanae (Froggatt, 1901) & - & $\mathrm{O}$ & & M63-XI \\
\hline Acizzia uncatoides (Ferris \& Klyver, 1932) & - & & & M49-XII; M50-II; M63-IV; M67-VIII \\
\hline Cacopsylla atlantica (Loginova, 1976) & - & & & A38-II; V59-II; M59-I; M59-IV \\
\hline \multicolumn{5}{|l|}{ Triozidae } \\
\hline Trioza chenopodii Reuter, 1876 & $\mathrm{O}$ & & & M6-V \\
\hline \multicolumn{5}{|l|}{$\begin{array}{l}\text { Orden Planipennia } \\
\text { Coniopterygidae }\end{array}$} \\
\hline Coniopteryx canariensis Monserrat, 2002 & $\mathrm{O}$ & $\mathrm{O}$ & $\mathrm{O}$ & M67-VII; M67-VIII \\
\hline
\end{tabular}




\begin{tabular}{|c|c|c|c|c|}
\hline Hemerobiidae & & & & \\
\hline Sympherobius pygmaeus (Rambur, 1842) & - & & & M67-VII; M67-VIII \\
\hline \multicolumn{5}{|l|}{$\begin{array}{l}\text { Orden Coleoptera } \\
\text { Anthicidae }\end{array}$} \\
\hline Anthicus crinitus Laferté, 1848 & $\mathrm{O}$ & & & ?17-VIII; W71-VIII; ?71-VIII \\
\hline \multicolumn{5}{|l|}{ Chrysomelidae } \\
\hline Psylliodes angusticeps Israelson, 1980 & $\mathrm{O}$ & & & 59-VII \\
\hline \multicolumn{5}{|l|}{ Cleridae } \\
\hline Denops canariensis Palm, 1978 & - & & & $\mathrm{X} 71-\mathrm{V}$ \\
\hline \multicolumn{5}{|l|}{ Curculionidae } \\
\hline Laparocerus n sp. & $\mathrm{O}$ & $\mathrm{O}$ & $\mathrm{O}$ & P19-V; P67-III; P18-IX; P19-IX; P66-III \\
\hline \multicolumn{5}{|l|}{ Hydrophilidae } \\
\hline Sphaeridium bipustulatum Fabricius, 1781 & - & - & & ?59-VII \\
\hline \multicolumn{5}{|l|}{ Languriidae } \\
\hline Setariola sericea Mulsant \& Rey, 1863 & - & & & YT59-IX \\
\hline \multicolumn{5}{|l|}{ Staphylinidae } \\
\hline Carpelimus flavomarginatus (Lindberg, 1953) & $\mathrm{O}$ & & & YT59-IX \\
\hline Neobisnius lathrobioides (Baudi, 1848) & $\mathrm{O}$ & & & YT59-IX \\
\hline $\begin{array}{l}\text { Spatulonthus longicornis piceicornis (Gridelli, 1920) } \\
\end{array}$ & - & - & & YT59-XI \\
\hline Sunius palmi (Franz, 1979) & $\mathrm{O}$ & & & TS71-XII \\
\hline \multicolumn{5}{|l|}{ Orden Trichoptera } \\
\hline Limnephilidae & O & & & \\
\hline Mesophylax aspersus Rambur, 1842 & - & & & A34-III; A34-IX; A37-III; A38-IX; A44-IX; A59-VII \\
\hline \multicolumn{5}{|l|}{ Orden Lepidoptera } \\
\hline Coccidiphila gerasimovi Danilevsky, 1950 & $\mathrm{O}$ & & & V29-VII \\
\hline \multicolumn{5}{|l|}{ Pyralidae } \\
\hline Botyodes diniasalis Walker, 1859 & - & & & L77-IX \\
\hline \multicolumn{5}{|l|}{ Orden Diptera } \\
\hline Agromyza brunnicosa Becker, 1908 & $\mathrm{O}$ & & & M59-XII \\
\hline Agromyza hierroensis Spencer, 1957 & O & & & M59-XII \\
\hline Liriomyza richteri Hering, 1927 & O & & & M59-XII \\
\hline Ophiomyia curvipalpis (Zetterstedt, 1848) & O & & & M6-VIII; M6-IX; YT6-VIII \\
\hline Campichoetidae & $\mathrm{O}$ & & & \\
\hline Campichoeta sp. & - & & & M59-XII \\
\hline \multicolumn{5}{|l|}{ Chloropidae } \\
\hline Polyodaspis sulcicollis (Meigen, 1838) & - & - & & YT63-IX \\
\hline \multicolumn{5}{|l|}{ Conopidae } \\
\hline Thecophora sp. & - & - & & YT63-IX \\
\hline \multicolumn{5}{|l|}{ Drosophilidae } \\
\hline Dettopsomyia nigrovittata (Malloch, 1924) & - & & & YT6-IX \\
\hline Scaptomyza adusta (Loew, 1862) & O & & & YT59-IX \\
\hline \multicolumn{5}{|l|}{ Ephydridae } \\
\hline Hydrellia maura Meigen, 1838 & - & & & YT59-IX \\
\hline \multicolumn{5}{|l|}{ Mycetophilidae } \\
\hline Docosia gilvipes (Haliday, 1856) & - & $\mathrm{O}$ & & M59-XII \\
\hline
\end{tabular}




\begin{tabular}{|c|c|c|c|c|}
\hline Leia beckeri Landrock, 1940 & $\mathrm{O}$ & & & M59-XII \\
\hline \multicolumn{5}{|l|}{ Phoridae } \\
\hline Conicera tibialis Schmitz, 1925 & $\mathrm{O}$ & & & YT59-IX; M59-XII \\
\hline Diplonerva funebris (Meigen,1830) & - & & & YT6-VIII; TC49-X; YT63-IX \\
\hline Megaselia albicaudata (Wood, 1910) & $\mathrm{O}$ & & & M59-XII \\
\hline Megaselia angusta (Wood, 1909) & $?$ & $?$ & & YT63-IX \\
\hline Megaselia apozona Schmitz, 1936 & $\mathrm{O}$ & & & M59-XII \\
\hline Megaselia basispinata (Lundbeck, 1920) & $\mathrm{O}$ & & & M59-XII \\
\hline Megaselia brevicostalis (Wood, 1910) & $\mathrm{O}$ & & & M59-XII \\
\hline Megaselia capronata (Schmitz, 1940) & 0 & 0 & & YT63-IX \\
\hline Megaselia cirratula/critinicauda-complex sp. n. & $\mathrm{O}$ & $\mathrm{O}$ & 0 & M59-XII \\
\hline Megaselia curtineura/innocens-complex sp. n. & 0 & 0 & 0 & TC49-VIII; TC49-IX \\
\hline Megaselia hirticaudata (Wood, 1910) & $\mathrm{O}$ & $O$ & & YT63-IX \\
\hline Megaselia hirtiventris (Wood, 1909) cf. & 0 & 0 & $?$ & M59-XII \\
\hline Megaselia longiseta (Wood, 1909) & $\mathrm{O}$ & $O$ & & M59-XII \\
\hline Metopina gr. heselhausi/oligoneura sp. $\mathrm{n}$. & $O$ & $O$ & $?$ & YT6-VIII; YT59-IX \\
\hline Metopina n.sp. & $\mathrm{O}$ & $\mathrm{O}$ & $\mathrm{O}$ & YT63-IX \\
\hline \multicolumn{5}{|l|}{ Psychodidae } \\
\hline Phlebotomus sp. & ○ & & & M6-IX \\
\hline Psychoda albipennis Zetterstedt, 1850 & $\mathrm{O}$ & $O$ & & M6-VIII \\
\hline Psychoda setigera Tonnoir, 1922 & $\mathrm{O}$ & $\mathrm{O}$ & & M6-IX; YT59-IX \\
\hline Sergentomyia minuta (Rondani, 1843) & $\boldsymbol{0}$ & & & YT63-VIII \\
\hline \multicolumn{5}{|l|}{ Sarcophagidae } \\
\hline Bercaea africa (Wiedemann, 1824) & $\mathrm{O}$ & & & TC49-VIII; YT59-IX; YT63-VIII \\
\hline Blaesoxipha lapidosa (Pape,1996) & $\mathbf{0}$ & $\mathrm{O}$ & & M6-VIII; YT6-VIII; YT6-IX \\
\hline Curranea tibialis (Macquart, 1850) & - & - & & YT6-VIII; TC49-X \\
\hline Heteronychia ferox Villeneuve, $1908 \mathrm{cf}$. & $?$ & $?$ & $?$ & YT59-IX \\
\hline Miltogramma aurifrons Dofur, 1850 & - & & & M6-VIII \\
\hline \multicolumn{5}{|l|}{ Sciaridae } \\
\hline Bradysia nomica Mohrig \& Röschmann, 1996 & $\mathrm{O}$ & & & M59-XII \\
\hline Bradysia triviatta (Staeger, 1840) & O & & & M6-VIII; M6-IX; M59-XII; YT63-VIII; YT63-IX \\
\hline Bradysiopsis n.sp. & - & - & 0 & M59-XII \\
\hline Corynoptera dentiforceps (Bukowski \& Lengersdorf, 1936) & $\mathrm{O}$ & & & YT6-VIII \\
\hline Corynoptera praeparvula Mohrig \& Krivosheina, 1983 & O & & & M59-XII \\
\hline Cratyna symplecta (Rudzinski, 1991) & - & & & M6-IX; YT63-VIII \\
\hline Moehnia erema Pritchard, 1960 & - & & & M6-IX \\
\hline Scaptosciara subarmata Mohrig \& Mamaev, 1983 & $O$ & & & M59-XII \\
\hline \multicolumn{5}{|l|}{ Sphaeroceridae } \\
\hline Chespiritos pervadens Rohácek \& Buck, sp. n. en prensa & - & - & $O$ & M6-VIII; YT59-IX \\
\hline \begin{tabular}{l|l} 
Coproica ferruginata (Stenhammar, 1854) \\
\end{tabular} & $\mathrm{O}$ & & & M6-VIII; YT6-VIII; YT6-IX \\
\hline Elachisoma aterrimum (Haliday, 1833) & - & & & M6-IX \\
\hline Opalimosina mirabilis (Collin, 1902) & - & & & YT63-VIII \\
\hline Phthitia plumosula (Rondani, 1880) & - & & & M6-VIII; M59-XII; YT59-IX \\
\hline Pullimosina heteroneura (Haliday, 1836) & - & & & M6-VIII; YT6-VIII \\
\hline \multicolumn{5}{|l|}{ Tachinidae } \\
\hline Aplomyia confinis (Fallén, 1820) & - & & & M6-VIII; YT6-VIII \\
\hline Ceracia mucronifera Rondani, 1865 & - & & & M6-VIII; M6-IX; YT6-VIII; YT6-IX \\
\hline Cylindromyia brassicaria (Fabricius, 1775) & - & & & M6-IX \\
\hline Exorista kugleri Mesnil, 1960 & $\mathrm{O}$ & & & YT6-IX \\
\hline
\end{tabular}




\begin{tabular}{|c|c|c|c|c|}
\hline Trixoscelididae & & & & \\
\hline Trixoscelis n.sp. & $\mathrm{O}$ & $\mathrm{O}$ & $\mathrm{O}$ & $\begin{array}{l}\text { YT6-VIII; M6-VIII; M6-IX; TC49-IX; YT63-IX; } \\
\text { YT63-VIII }\end{array}$ \\
\hline \multicolumn{5}{|l|}{ Orden Hymenoptera } \\
\hline Aphelinus chaonia Walker, 1839 & O & $\mathrm{O}$ & & V31-III; V48-II \\
\hline \multicolumn{5}{|l|}{ Braconidae } \\
\hline Agathis tibialis Nees, 1814 & - & - & & M67-VII; M67-VIII \\
\hline Aleiodes borealis (Thomson, 1891) & ○ & O & & M67-VIII; M67-IX \\
\hline Apanteles sp. & $?$ & & & $\begin{array}{l}\text { M6-VIII; M6-IX; M59-XII; YT59-IX; M63-VIII; } \\
\text { YT63-VIII }\end{array}$ \\
\hline Aspilotes sp. & - & - & & YT59-IX; M59-XII; YT63-VIII \\
\hline Blacus diversicornis (Nees, 1834) & - & & & YT59-IX \\
\hline Blacus imitator Papp, 1985 cf. & - & O & $?$ & M6-VIII \\
\hline Bracon nigricans Szepligeti, 1901 & $\mathrm{O}$ & $\mathrm{O}$ & & M6-IX; YT59-IX; M67-VIII \\
\hline Chelonus sp. & - & & & M67-VII \\
\hline Diristrema sp. & - & - & & M49-XI; M49-XII; M63-VIII \\
\hline Hormius sp. & - & & & M6-VIII; M59-XII \\
\hline Microplitis sp. & - & $?$ & & M49-XI; M67-IX \\
\hline Pambolus sp. & - & & & B77-IX \\
\hline Protapanteles sp. & - & • & & $\begin{array}{l}\text { M6-VIII; M6-IX; M59-XII; M63-VIII; YT63-VIII; } \\
\text { M67-VIII }\end{array}$ \\
\hline \multicolumn{5}{|l|}{ Chalcididae } \\
\hline Proconura nigripes (Fonscolombe, 1832) & $O$ & & & YT63-VIII \\
\hline \multicolumn{5}{|l|}{ Chrysididae } \\
\hline Chrysis succinta Linnaeus, $1767 \mathrm{cf}$. & $\mathrm{O}$ & $\mathrm{O}$ & & YT6-VIII \\
\hline Diapriidae & $\mathrm{O}$ & $\mathrm{O}$ & & M6-IX; M49-XII; YT59-IX; M59-XII; YT63-IX \\
\hline \multicolumn{5}{|l|}{ Encyrtidae } \\
\hline Charitopus sp. & - & & & V65-VII \\
\hline Ectroma koponeni Trjapitzin, 1989 & - & O & & V9-VII \\
\hline Trechnites fuscitarsis Thomson, 1876 & - & - & & V63-IX; V52-IX; V63-III \\
\hline \multicolumn{5}{|l|}{ Eulophidae } \\
\hline Aprostocetus sp. n. A & $\mathrm{O}$ & $\mathrm{O}$ & $\mathrm{O}$ & V31-III; V31-VI; V41-I; V46-VII; V71-III \\
\hline Aprostocetus sp. n. B & $O$ & $\mathrm{O}$ & $O$ & V46-VII \\
\hline Aprostocetus sp. n. C & $\mathrm{O}$ & $\mathrm{O}$ & $\mathrm{O}$ & V60-VI; V71-V \\
\hline Aprostocetus sp. n. D & O & O & $\mathrm{O}$ & V43-VII \\
\hline Aprostocetus sp. n. E & $\mathrm{O}$ & $\mathrm{O}$ & $\mathrm{O}$ & V31-V \\
\hline Cirrospilus lyncus Walker, 1838 & O & $\mathrm{O}$ & & V57-IX \\
\hline Elachertus fenestratus (Nees, 1834) & $\mathrm{O}$ & $\mathrm{O}$ & & YT59-IX \\
\hline Platyplectrus bouceki (Erdös, 1966) & - & - & & V52-IX \\
\hline Pnigalio agraules (Walker, 1839) & $\mathrm{O}$ & $\mathrm{O}$ & & V21-V; V30-V; V67-VI \\
\hline \multicolumn{5}{|l|}{ Eupelmidae } \\
\hline Anastatus giraudi (Ruschka, 1921) & - & - & & M67-VIII \\
\hline Calymmochilus subnubilus (Walker, 1872) & $\bullet$ & - & & M6-VIII; M6-IX; V52-IX \\
\hline Eusandulum merceti (Bolivar, 1926) & - & - & & V5-VI \\
\hline \multicolumn{5}{|l|}{ Ichneumonidae } \\
\hline Barichneumon canariensis Hellen, 1949 & - & & & M6-VIII; M67-VIII; M67-IX \\
\hline Campoplex faunus Gravenhorst, 1829 & $\mathrm{O}$ & & & YT59-IX \\
\hline Campoplex puncticollis (Hellen, 1949) & $\mathrm{O}$ & & & M67-VII; M67-VIII \\
\hline Casinaria sp. & - & - & & YT6-VIII; M6-VIII; M6-IX; YT63-IX \\
\hline
\end{tabular}




\begin{tabular}{|c|c|c|c|c|}
\hline Coelichneumon cabrerai (Berthoumieu, 1903) & 0 & & & M6-IX; YT59-IX; M67-VIII \\
\hline Cryptinae (Subfam.) gen. sp. 1 & $?$ & $?$ & $?$ & M59-XII \\
\hline Cryptinae (Subfam.) gen. sp. 2 & $?$ & $?$ & $?$ & M67-VIII \\
\hline Cryptus spinosus Gravenhorst, 1829 & O & O & & M6-VIII; M6-IX \\
\hline Cymodusa ancilla (Seyrig, 1927) & - & & & M59-XII \\
\hline Diadegma filicorne (Horstmann, 1980) & O & & & M6-IX \\
\hline Diadegma mollipla (Holmgren, 1868) & $\mathrm{O}$ & & & YT63-VIII \\
\hline Diadegma semiclausum (Hellen, 1949) & O & & & M67-IX \\
\hline Dicaelotus sp. & - & & & M59-XII \\
\hline Dichrogaster tenerifae (Hellen, 1949) & - & & & M67-VIII; M67-IX \\
\hline Eparces sp. & - & ○ & & YT59-IX \\
\hline Heterischnus rufithorax (Berthoumieu, 1903) & - & & & M67-VIII \\
\hline Lissonota bivittata Gravenhorst, 1829 & - & $\mathrm{O}$ & & M67-IX \\
\hline Ophion luteus (Linnaeus, 1758) & - & & & M59-XII \\
\hline Ophion obscuratus Fabricius, 1798 & - & & & V69-IV \\
\hline Scambus buoliana (Hartig, 1838) & $\mathrm{O}$ & O & & M49-XII \\
\hline Temelucha decorata (Gravenhorst, 1829) & $\mathrm{O}$ & & & M67-VII; M67-VIII; M67-IX \\
\hline Trychosis legator (Thunberg, 1822) & - & & & M67-VIII \\
\hline $\begin{array}{l}\text { Perilampidae } \\
\end{array}$ & $\mathrm{O}$ & $\mathrm{O}$ & & \\
\hline Perilampus aeneus (Rossius, 1790) cf. & - & - & $?$ & M67-VIII \\
\hline Platygastridae & O & & & \\
\hline Amblyaspis prorsa (Walker, 1835) & $\bullet$ & $\bullet$ & & $\begin{array}{l}\text { M6-VIII; M6-IX; YT6-VIII; YT6-IX; M49-XII; } \\
\text { YT59-IX; YT63-IX }\end{array}$ \\
\hline Iphitrachelus gracilis Masner, 1957 & - & - & & YT63-IX \\
\hline Leptacis vlugi Buhl, 1997 & - & - & & M59-XII; YT63-IX \\
\hline Platygaster tenerifensis Buhl, 2001 & - & & & M6-IX \\
\hline Synopeas blascoi Buhl, 1998 cf. & - & $\bullet$ & $?$ & YT63-IX; M67-VII; M67-VIII \\
\hline Synopeas ciliatus Thomson, 1859 & - & - & & M59-XII \\
\hline \multicolumn{5}{|l|}{ Pompilidae } \\
\hline Agenioideus simonthomasi Wolf, 1978 & - & & & YT63-VIII; M67-VIII \\
\hline Proctotrupidae & $\mathrm{O}$ & $\mathrm{O}$ & & YT59-IX \\
\hline \multicolumn{5}{|l|}{ Pteromalidae } \\
\hline Callitula bicolor Spinola, 1811 & - & & & YT59-IX \\
\hline Chlorocytus spicatus (Walker, 1835) cf. & - & - & $?$ & V67-VI \\
\hline Dibrachys cavus (Walker, 1835) & - & - & & M67-VII; M67-VIII; M67-IX; TC67-VIII \\
\hline Eunotus merceti Masi, 1931 & - & O & & M6-VIII; M63-VIII; M67-VIII; M67-X \\
\hline Mesopolobus aspilus (Walker, 1835) & $\mathrm{O}$ & O & & V6-III; V75-III \\
\hline Mesopolobus incultus (Walker, 1834) cf. & O & O & $?$ & M49-XII; M67-VIII \\
\hline Metastenus concinnus Walker, 1834 & - & - & & M6-IX \\
\hline Nasonia vitripennis (Walker, 1836) & - & - & & F50-VI; F50-VII; F69-VI \\
\hline Pachyneuron muscarum (Linnaeus, 1758) & O & O & & $\begin{array}{l}\text { V5-V; M63-VIII; YT63-VIII; YT63-IX; M67-VII; } \\
\text { M67-VIII; M67-IX; V71-XII }\end{array}$ \\
\hline Pteromalus brachygaster (Graham, 1969) cf. & O & $O$ & $?$ & V67-VI; V67-VII; V69-VI \\
\hline Spalangia subpunctata Förster, 1850 & $\mathrm{O}$ & O & & M59-XII \\
\hline Spalangiopelta procera Graham, 1966 & - & - & & M6-IX \\
\hline Systasis encyrtoides Walker, 1834 cf. & $\mathrm{O}$ & $?$ & $?$ & V5-V; V6-V; V31-III; V41-III; V54-V; V71-V; V77-V \\
\hline \multicolumn{5}{|l|}{ Scelionidae } \\
\hline Eremioscelio cynoides Priesner, 1951 & - & - & & M67-VIII \\
\hline Gryon misellum Haliday, 1833 & - & ? & & M6-VIII \\
\hline Siricidae & $\mathrm{O}$ & & & \\
\hline Sirex noctilio Fabricius, 1793 & - & & & $? 77-\mathrm{X}$ \\
\hline
\end{tabular}




\begin{tabular}{|c|c|c|c|c|}
\hline Sphecidae & & & & \\
\hline Solierella canariensis Saunders, 1904 & - & & & YT63-IX \\
\hline \multicolumn{5}{|l|}{ Tetracampidae } \\
\hline Epicleurus temenus (Walker, 1839) cf. & $\mathrm{O}$ & $?$ & $?$ & M6-IX; YT63-IX \\
\hline \multicolumn{5}{|l|}{ Torymidae } \\
\hline Podagrionella lichtensteini (Picard, 1933) & $\bullet$ & - & & M49-XII \\
\hline Pseudotorymus sp. & $\bullet$ & - & & V54-V; V71-V; V77-I; V77-V \\
\hline Torymus sp. & - & & & V77-I \\
\hline
\end{tabular}

